\title{
Gibbs States, Algebraic Dynamics and Generalized Riesz Systems
}

\author{
F. Bagarello $o^{1,2} \cdot$ H. Inoue ${ }^{3} \cdot$ C. Trapani ${ }^{4}$
}

Received: 28 April 2020 / Accepted: 10 September 2020 / Published online: 13 October 2020

(c) The Author(s) 2020

\begin{abstract}
In PT-quantum mechanics the generator of the dynamics of a physical system is not necessarily a self-adjoint Hamiltonian. It is now clear that this choice does not prevent to get a unitary time evolution and a real spectrum of the Hamiltonian, even if, most of the times, one is forced to deal with biorthogonal sets rather than with on orthonormal basis of eigenvectors. In this paper we consider some extended versions of the Heisenberg algebraic dynamics and we relate this analysis to some generalized version of Gibbs states and to their related KMS-like conditions. We also discuss some preliminary aspects of the Tomita-Takesaki theory in our context.
\end{abstract}

Keywords Gibbs states · Non-Hermitian Hamiltonians · Biorthogonal sets of vectors · Tomita-Takesaki theory

Communicated by Sergey Naboko.

Data sharing not applicable to this article as no datasets were generated or analysed during the current study.

$凶$ C. Trapani

camillo.trapani@unipa.it

F. Bagarello

fabio.bagarello@unipa.it

https://www1.unipa.it/fabio.bagarello

H. Inoue

h-inoue@daiichi-cps.ac.jp

1 Dipartimento di Ingegneria, Università di Palermo, 90128 Palermo, Italy

2 Sezione di Napoli, INFN, L'Aquila, Italy

3 Center for Advancing Pharmaceutical Education, Daiichi University of Pharmacy, 22-1

Tamagawa-cho, Minami-ku, Fukuoka 815-8511, Japan

4 Dipartimento di Matematica e Informatica, Università di Palermo, 90123 Palermo, Italy

Birkhäuser 


\section{Introduction}

In the past 25 years or so it has become clearer and clearer that the role of selfadjointness of the observables of some given microscopic system can be, sometimes, relaxed, without modifying the essential benefits of dealing with, for instance, a selfadjoint Hamiltonian. In fact, we can still find real eigenvalues, a unitary time evolution and a preserved probability even if the requirement of the Hamiltonian being selfadjoint is replaced by some milder assumption, like in PT- or in pseudo-hermitian quantum mechanics. We refer to [1-5] for some references on these approaches, both from a more physical point of view and from their mathematical consequences.

Considering a non-selfadjoint Hamiltonian $H \neq H^{*}$ may lead to the appearance of new and often unpleasant features; for instance, the set $\left\{\varphi_{n}\right\}$ of eigenstates of $H$, if any, in general is no longer an orthonormal system, but this set $\left\{\varphi_{n}\right\}$ and the set $\left\{\psi_{n}\right\}$ of the eigenstates of $H^{*}$ turn out to be biorthogonal i.e., $\left(\varphi_{n} \mid \psi_{m}\right)=\delta_{n, m}$. Also, in concrete examples they are not bases for the Hilbert space $\mathcal{H}$ where the model is defined, but they may still be complete in $\mathcal{H}$. This is the reason why the notion of $\mathcal{D}$-quasi bases was proposed in [6].

This concept can be thought as a suitable extension of Riesz biorthogonal bases, and similar biorthogonal sets are found in several concrete physical applications, playing often the role that in the traditional setup is played by orthonormal bases (ONB). In recent papers many other extensions of Riesz bases, mostly involving unbounded operators, have also been considered. In particular we mention generalized Riesz systems introduced by one of us (H.I) and analyzed in a series of papers [714]). For other studies on extensions of Riesz bases or on generalizations to different environments (Krein spaces, Rigged Hilbert spaces) we refer to [15-17].

In [18] the role of similar biorthogonal sets, in particular Riesz bases, in the analysis of Gibbs states, KMS condition and algebraic Heisenberg dynamics was first considered. More recently a similar analysis has been carried out by other authors (see, e.g. [19]). Here we want to give our contribution to this line of research, by using the biorthogonal sets originated by generalized Riesz systems.

The paper is organized as follows: in the next section we give some preliminaries. In Sect. 3 we propose our definition of Gibbs state defined by generalized Riesz systems, when the dynamics is driven by a self-adjoint operator $H_{0}$. The natural settings which we will adopt is the $O^{*}$-algebra $\mathcal{L}^{\dagger}(\mathcal{D})$, where $\mathcal{D}$ is a dense subspace of $\mathcal{H}$, [20-22]. This will appear to be a good choice, due to the fact that the operators appearing in our analysis are mostly unbounded. In Sect. 4 we will consider possible definitions of the algebraic dynamics for non self-adjoint Hamiltonians, and then we will consider how these dynamics are related to the generalized Gibbs states introduced first, and the KMS-like relations which arise from this construction. In Sect. 5 we will propose a preliminary analysis of the Tomita-Takesaki modular theory in our context, while our conclusions are given in Sect. 6. 


\section{Preliminaries}

In this section we review the basic definitions and results on generalized Riesz systems and $O^{*}$-algebras needed in this paper.

Definition 2.1 A sequence $\left\{\varphi_{n}\right\}$ in a Hilbert space $\mathcal{H}$ with inner product $(\cdot \mid \cdot)$ is called a generalized Riesz system if there exist an $\operatorname{ONB}\left\{f_{n}\right\}$ in $\mathcal{H}$ and a densely defined closed operator $T$ in $\mathcal{H}$ with densely defined inverse, such that $\left\{f_{n}\right\} \subset D(T) \cap D\left(\left(T^{-1}\right)^{*}\right)$ and $T f_{n}=\varphi_{n}, n=0,1, \ldots$ Such a $\left(\left\{f_{n}\right\}, T\right)$ is called a constructing pair for $\left\{\varphi_{n}\right\}$ and $T$ is called a constructing operator for $\left\{\varphi_{n}\right\}$.

Suppose that $\left(\left\{\varphi_{n}\right\},\left\{\psi_{n}\right\}\right)$ is a biorthogonal pair such that $\left\{\varphi_{n}\right\}$ be a generalized Riesz system with a constructing pair $\left(\left\{f_{n}\right\}, T\right)$. Then putting $\psi_{n}^{T}=\left(T^{-1}\right)^{*} f_{n}, n=$ $0,1, \ldots,\left\{\varphi_{n}\right\}$ and $\left\{\psi_{n}^{T}\right\}$ are biorthogonal sequences, that is, $\left(\varphi_{n} \mid \psi_{m}^{T}\right)=\delta_{n m}, n, m=$ $0,1, \ldots$. If $\psi_{n}^{T}=\psi_{n}, n=0,1, \ldots$, then $\left\{\psi_{n}\right\}$ is a generalized Riesz system with a constructing pair $\left(\left\{f_{n}\right\},\left(T^{-1}\right)^{*}\right)$. But, the equality $\psi_{n}^{T}=\psi_{n}, n=0,1, \ldots$ does not necessarily hold. If this equality holds, then $T$ is called natural and $\left(\left\{f_{n}\right\}, T\right)$ is called natural constructing pair.

Let $\mathcal{D}$ be a dense subspace in $\mathcal{H}$. We denote by $\mathcal{L}^{\dagger}(\mathcal{D}, \mathcal{H})$ the set of all closable linear operators $X$ in $\mathcal{H}$ such that $D(X)=\mathcal{D}$ and $D\left(X^{*}\right) \supset \mathcal{D}$. As usual we put, for $X \in \mathcal{L}^{\dagger}(\mathcal{D}, \mathcal{H}), X^{\dagger}:=X^{*}\lceil\mathcal{D}$. Let

$$
\begin{aligned}
\mathcal{L}(\mathcal{D}) & =\left\{X \in \mathcal{L}^{\dagger}(\mathcal{D}, \mathcal{H}) ; X \mathcal{D} \subset \mathcal{D}\right\} \\
\mathcal{L}^{\dagger}(\mathcal{D}) & =\left\{X \in \mathcal{L}(\mathcal{D}) ; X^{*} \mathcal{D} \subset \mathcal{D}\right\}
\end{aligned}
$$

Then $\mathcal{L}(\mathcal{D})$ is an algebra with the usual operations: $X+Y, \alpha X$ and $X Y$, and $\mathcal{L}^{\dagger}(\mathcal{D})$ is a $*$-algebra with the involution $X \rightarrow X^{\dagger}:=X^{*}\left\lceil\mathcal{D}\right.$, inherited by $\mathcal{L}^{\dagger}(\mathcal{D}, \mathcal{H})$. A *-subalgebra $\mathcal{M}$ of $\mathcal{L}^{\dagger}(\mathcal{D})$ is said to be an $O^{*}$-algebra on $\mathcal{D}$ in $\mathcal{H}$. Here we assume that $\mathcal{M}$ has the identity operator $I$. A locally convex topology defined by a family $\left\{\|\cdot\|_{X} ; X \in \mathcal{M}\right\}$ of seminorms: $\|\xi\|_{X}:=\|X \xi\|, \xi \in \mathcal{D}$ is called the graph topology on $\mathcal{D}$ and denoted by $t_{\mathcal{M}}$. If the locally convex space $\mathcal{D}\left[t_{\mathcal{M}}\right]$ is complete, then $\mathcal{M}$ is called closed and it is shown that $\mathcal{M}$ is closed if and only if $\mathcal{D}=\bigcap_{X \in \mathcal{M}} D(\bar{X})$. If $\mathcal{D}=\bigcap_{X \in \mathcal{M}} D\left(X^{*}\right)$, then $\mathcal{M}$ is called self-adjoint. Next we define a weak commutant of $\mathcal{M}$ as follows:

$$
\mathcal{M}_{w}^{\prime}:=\left\{C \in B(\mathcal{H}) ;(C X \xi \mid \eta)=\left(C \xi \mid X^{\dagger} \eta\right) \text { for all } X \in \mathcal{M} \text { and } \xi, \eta \in \mathcal{D}\right\}
$$

where $B(\mathcal{H})$ is the $C^{*}$-algebra of all bounded linear operators on $\mathcal{H}$. Then $\mathcal{M}_{w}^{\prime}$ is a weakly closed $*$-invariant subspace of $B(\mathcal{H})$, but it is not necessarily an algebra. If $\mathcal{M}$ is self-adjoint, then $\mathcal{M}_{w}^{\prime}$ is a von Neumann algebra on $\mathcal{H}$ satisfying $\mathcal{M}_{w}^{\prime} \mathcal{D} \subset \mathcal{D}$. Furthermore, we see that $\mathcal{L}^{\dagger}(\mathcal{D})_{w}^{\prime}=\mathbb{C} I$. We define some topologies on $\mathcal{M}$. For any $\xi, \eta \in \mathcal{D}$ we put $p_{\xi, \eta}(X):=|(X \xi \mid \eta)|, p_{\xi}(X):=\|X \xi\|, X \in \mathcal{L}^{\dagger}(\mathcal{D})$. The locally convex topology on $\mathcal{L}^{\dagger}(\mathcal{D})$ defined by the family $\left\{p_{\xi, \eta}(\cdot) ; \xi, \eta \in \mathcal{D}\right\}$ (resp. $\left\{p_{\xi}(\cdot) ; \xi \in \mathcal{D}\right\}$ ) of seminorms on $\mathcal{L}^{\dagger}(\mathcal{D})$ is called the weak (resp. strong) topology, and the induced topology of the weak (resp. strong) topology on $\mathcal{M}$ is called the weak (resp. strong) topology on $\mathcal{M}$. For any $Y \in \mathcal{M}$ and $\xi \in \mathcal{D}$ we define a seminorm on 
$\mathcal{M}$ by

$$
p_{\xi, Y}(X):=\|Y X \xi\|, \quad X \in \mathcal{M}
$$

The locally convex topology on $\mathcal{M}$ defined by the family $\left\{P_{\xi, Y}(\cdot) ; \xi \in \mathcal{D}, Y \in \mathcal{M}\right\}$ is called the quasi-strong topology on $\mathcal{M}$. A linear functional $\omega$ on $\mathcal{M}$ is called positive if $\omega\left(X^{\dagger} X\right) \geqq 0$ for all $X \in \mathcal{M}$, and a positive linear functional $\omega$ on $\mathcal{M}$ is a state if $\omega(I)=1$. A $(*)$-isomorphism of $\mathcal{M}$ onto $\mathcal{M}$ is called a (*)-automorphism of $\mathcal{M}$ and $\left\{\alpha_{t}\right\}_{t \in \mathbb{R}}$ is called a one-parameter group of $(*)$-automorphisms of $\mathcal{M}$ if $\alpha_{0}(X)=X$ and $\alpha_{s+t}(X)=\alpha_{s}\left(\alpha_{t}(X)\right)$ for all $X \in \mathcal{M}$. A one-parameter group $\left\{\alpha_{t}\right\}_{t \in \mathbb{R}}$ of automorphisms of $\mathcal{M}$ is weakly (resp. strongly, quasi-strongly) continuous if $\lim _{t \rightarrow 0} \alpha_{t}(X)=X$ for any $X \in \mathcal{M}$ under the weak (resp. strong, quasi-strong) topology. An operator $H$ in $\mathcal{L}^{\dagger}(\mathcal{D})$ is called a weak (resp. strong, quasi-strong) generator for $\left\{\alpha_{t}\right\}_{t \in \mathbb{R}}$ if $\lim _{t \rightarrow 0} \frac{\alpha_{t}(X)-X}{t}=i[H, X]$ under the weak (resp. strong, quasi-strong) topology. For $O^{*}$-algebras refer to [23].

\section{Gibbs States Defined by Generalized Riesz Systems}

Throughout this section let $\left\{\varphi_{n}\right\}$ be a generalized Riesz system in a Hilbert space $\mathcal{H}$ with a constructing pair $\left(\left\{f_{n}\right\}, T\right)$ and $\lambda_{n}>0, n=0,1, \ldots$, such that $\left\{e^{-\frac{\beta}{2} \lambda_{n}}\right\} \in \ell^{1}$, for some $\beta>0$. In this section we shall define and investigate a Gibbs state $\omega_{\varphi}^{\beta}$ defined through $\left\{\varphi_{n}\right\}$ on the maximal $O^{*}$-algebra $\mathcal{L}^{\dagger}(\mathcal{D})$ on a dense subspace $\mathcal{D}$ in $\mathcal{H}$. We put

$$
H_{0}:=\sum_{n=0}^{\infty} \lambda_{n} f_{n} \otimes \bar{f}_{n},
$$

where, for $x, y \in \mathcal{H}$, the operator $x \otimes \bar{y}$ is defined by

$$
(x \otimes \bar{y}) \xi=(\xi \mid y) x, \quad \xi \in \mathcal{H} .
$$

Then $H_{0}$ is a non-singular positive self-adjoint operator in $\mathcal{H}$ such that

$$
H_{0} f_{n}=\lambda_{n} f_{n}, \quad n \in \mathbb{N}_{0}:=\mathbb{N} \cup\{0\},
$$

and it is called a standard hamiltonian for $\left\{f_{n}\right\}$.

Before entering in the main matter of the paper some comments are in order. Once $H_{0}$ and a generalized Riesz system $\left\{\varphi_{n}\right\}$ with constructing pair $\left(\left\{f_{n}\right\}, T\right)$ are given, one can define an operator $H$ on the linear span $D_{\varphi}$ of $\left\{\varphi_{n}\right\}$ by putting $H \varphi_{n}=\lambda_{n} \varphi_{n} ; n \in$ $\mathbb{N}_{0}$ and extending by linearity to $D_{\varphi}$. Since $D_{\varphi}$ needs not be dense in $\mathcal{H}$, it is natural to consider $H$ as an operator acting in $\mathcal{H}_{\varphi}$, the closure of $D_{\varphi}$ in $\mathcal{H}$. It is then natural to write $H \varphi_{n}=H T f_{n}=\lambda_{n} \varphi_{n}=T H_{0} f_{n}, n \in \mathbb{N}$, which looks like an intertwining (or, better, when $\mathrm{T}$ is invertible, a similarity) condition for $H$ and $\mathcal{H}_{0}$, as discussed in [18] for Riesz bases. Similarity is a quite strong condition in particular when considering the spectrum of the involved operators or trying to get a functional calculus. We will 
not pursue this approach here because it doesn't fit with the general situation we are considering.

Lemma 3.1 Let $\mathcal{D}$ be a dense subspace in $\mathcal{H}$. Suppose that

$$
e^{-\frac{\beta}{2} H_{0}} \mathcal{H} \subset \mathcal{D}
$$

Then $X e^{-\beta H_{0}}$ is trace class on $\mathcal{H}$, for all $X \in \mathcal{L}^{\dagger}(\mathcal{D}, \mathcal{H})$.

Proof Take an arbitrary $X \in \mathcal{L}^{\dagger}(\mathcal{D}, \mathcal{H})$. Since $e^{-\frac{\beta}{2} H_{0}} \mathcal{H} \subset \mathcal{D}$, we have

$$
D\left(X e^{-\frac{\beta}{2} H_{0}}\right)=\mathcal{H}
$$

Thus, $X e^{-\frac{\beta}{2} H_{0}}$ is an everywhere defined operator on $\mathcal{H}$ and it is simple to show that it is closable. Therefore $X e^{-\frac{\beta}{2} H_{0}}$ is a closed operator in $\mathcal{H}$. By the closed graph theorem $X e^{-\frac{\beta}{2} H_{0}}$ is a bounded operator on $\mathcal{H}$ and since

$$
X e^{-\beta H_{0}}=\left(X e^{-\frac{\beta}{2} H_{0}}\right) e^{-\frac{\beta}{2} H_{0}},
$$

we have $X e^{-\beta H_{0}}$ is trace class. This completes the proof.

We remark that any subspace $\mathcal{D}$ in $\mathcal{H}$ satisfying (3.1) is dense in $\mathcal{H}$ since it contains the ONB $\left\{f_{n}\right\}$ in $\mathcal{H}$, and $D^{\infty}\left(H_{0}\right):=\bigcap_{n \in \mathbb{N}} D\left(H_{0}^{n}\right)$ is a subspace in $\mathcal{H}$ satisfying (3.1).

Under assumption (3.1) we can introduce a state on $\mathcal{L}^{\dagger}(\mathcal{D})$ by

$$
\omega_{f}^{\beta}(X):=\frac{1}{Z_{f}} \sum_{n=0}^{\infty} e^{-\beta \lambda_{n}}\left(X f_{n} \mid f_{n}\right), \quad X \in \mathcal{L}^{\dagger}(\mathcal{D})
$$

where $Z_{f}:=\sum_{n=0}^{\infty} e^{-\beta \lambda_{n}}$. Indeed, by Lemma 3.1 we have

$$
\begin{aligned}
\operatorname{tr}\left(X e^{-\beta H_{0}}\right) & =\sum_{n=0}^{\infty}\left(X e^{\beta H_{0}} f_{n} \mid f_{n}\right) \\
& =\sum_{n=0}^{\infty} e^{-\beta \lambda_{n}}\left(X f_{n} \mid f_{n}\right),
\end{aligned}
$$

for all $X \in \mathcal{L}^{\dagger}(\mathcal{D})$, and hence $\omega_{f}^{\beta}$ is a state on $\mathcal{L}^{\dagger}(\mathcal{D})$, and it is called a Gibbs state on $\mathcal{L}^{\dagger}(\mathcal{D})$ for the ONB $\left\{f_{n}\right\}$. We formally define a Gibbs state $\omega_{\varphi}^{\beta}$ on $\mathcal{L}^{\dagger}(\mathcal{D})$ for the generalized Riesz system $\left\{\varphi_{n}\right\}$ by

$$
\omega_{\varphi}^{\beta}(X):=\frac{1}{Z_{\varphi}} \sum_{n=0}^{\infty} e^{-\beta \lambda_{n}}\left(X \varphi_{n} \mid \varphi_{n}\right), \quad X \in \mathcal{L}^{\dagger}(\mathcal{D})
$$


where $Z_{\varphi}:=\sum_{n=0}^{\infty} e^{-\beta \lambda_{n}}\left\|\varphi_{n}\right\|^{2}$. Conditions for that are discussed in [18]. In what follows we will consider only generalized Riesz system $\left\{\varphi_{n}\right\}$ for which $Z_{\varphi}<\infty$.

We do not know whether $\omega_{\varphi}^{\beta}$ is a state on $\mathcal{L}^{\dagger}(\mathcal{D})$, namely, in particular, $\left|\omega_{\varphi}^{\beta}(X)\right|<\infty$ for all $X \in \mathcal{L}^{\dagger}(\mathcal{D})$. For that, we assume that a constructing pair $\left(\left\{f_{n}\right\}, T\right)$ for a generalized Riesz system $\left\{\varphi_{n}\right\}$ satisfies the following

Assumption 1 There exists a dense subspace $\mathcal{D}$ in $\mathcal{H}$ satisfying

(i) $e^{-\frac{\beta}{2} H_{0}} \mathcal{H} \subset \mathcal{D}$,

(ii) $\mathcal{D} \subset D(T) \cap D\left(T^{*}\right)$,

(iii) $T\left\lceil_{\mathcal{D}}\right.$ (the restriction of $T$ to $\left.\mathcal{D}\right) \in \mathcal{L}(\mathcal{D})$.

By (ii) in Assumption $1, T\left\lceil\mathcal{D} \in \mathcal{L}^{\dagger}(\mathcal{D}, \mathcal{H})\right.$. In the rest of the paper we will use the same symbol for $T, e^{-\frac{\beta}{2} H_{0}}$ and $e^{-\beta H_{0}}$, and for their restrictions to $\mathcal{D}$. Then we have the following

Theorem 3.2 Under Assumption 1, $\omega_{\varphi}^{\beta}$ is a faithful state on $\mathcal{L}^{\dagger}(\mathcal{D})$ and

$$
\begin{aligned}
\omega_{\varphi}^{\beta}(X) & =\frac{1}{Z_{\varphi}} \operatorname{tr}\left(T^{*} X T e^{-\beta H_{0}}\right) \\
& =\frac{1}{Z_{\varphi}} \operatorname{tr}\left(\left(T e^{-\frac{\beta}{2} H_{0}}\right)^{*} X\left(T e^{-\frac{\beta}{2} H_{0}}\right)\right)
\end{aligned}
$$

for all $X \in \mathcal{L}^{\dagger}(\mathcal{D})$.

Here a state $\omega$ on $\mathcal{L}^{\dagger}(\mathcal{D})$ is said to be faithful if $\omega\left(X^{\dagger} X\right)=0, X \in \mathcal{L}^{\dagger}(\mathcal{D})$, then $X=0$.

Proof Take an arbitrary $X \in \mathcal{L}^{\dagger}(\mathcal{D})$. Then, by Assumption $1, T^{*} X T \in \mathcal{L}^{\dagger}(\mathcal{D}, \mathcal{H})$ and by Lemma $3.1\left(T^{*} X T\right) e^{-\beta H_{0}}$ is trace class, which implies that

$$
\begin{aligned}
\frac{1}{Z_{\varphi}} \operatorname{tr}\left(T^{*} X T e^{-\beta H_{0}}\right) & =\frac{1}{Z_{\varphi}} \sum_{n=0}^{\infty}\left(T^{*} X T e^{-\beta H_{0}} f_{n} \mid f_{n}\right) \\
& =\frac{1}{Z_{\varphi}} \sum_{n=0}^{\infty} e^{-\beta \lambda_{n}}\left(T^{*} X T f_{n} \mid f_{n}\right) \\
& =\sum_{n=0}^{\infty} e^{-\beta \lambda_{n}}\left(X \varphi_{n} \mid \varphi_{n}\right)
\end{aligned}
$$

for all $X \in \mathcal{L}^{\dagger}(\mathcal{D})$. Hence $\omega_{\varphi}^{\beta}(X)=\frac{1}{Z_{\varphi}} \operatorname{tr}\left(T^{*} X T e^{-\beta H_{0}}\right)$ and it is a state on $\mathcal{L}^{\dagger}(\mathcal{D})$. Since $T$ and $T^{*}$ are non-singular; that is, $T^{-1}$ and $\left(T^{*}\right)^{-1}$ exist, we see that $\omega_{\varphi}^{\beta}$ is faithful. Furthermore, we have 


$$
\begin{aligned}
\omega_{\varphi}(X) & =\frac{1}{Z_{\varphi}} \operatorname{tr}\left(T^{*} X T e^{-\beta H_{0}}\right) \\
& =\frac{1}{Z_{\varphi}} \operatorname{tr}\left(\left(T^{*} X T e^{-\frac{\beta}{2} H_{0}}\right) e^{-\frac{\beta}{2} H_{0}}\right) \\
& =\frac{1}{Z_{\varphi}} \operatorname{tr}\left(e^{-\frac{\beta}{2} H_{0}}\left(T^{*} X T\right) e^{-\frac{\beta}{2} H_{0}}\right) \\
& =\frac{1}{Z_{\varphi}} \operatorname{tr}\left(\left(T e^{-\frac{\beta}{2} H_{0}}\right)^{*} X\left(T e^{-\frac{\beta}{2} H_{0}}\right)\right),
\end{aligned}
$$

for all $X \in \mathcal{L}^{\dagger}(\mathcal{D})$. This completes the proof.

Remark Clearly, $\omega_{\varphi}(X)=\frac{Z_{0}}{Z_{\varphi}} \omega_{0}\left(T^{*} X T\right)$, for every $X \in \mathcal{L}^{\dagger}(\mathcal{D})$, where $\omega_{0}(X)=$ $\frac{1}{Z_{0}} \operatorname{tr}\left(X e^{-\beta H_{0}}\right)$ and $Z_{0}=\sum_{n=0}^{\infty} e^{-\beta \lambda_{n}}$, as usually introduced in the literature when in presence of a self-adjoint Hamiltonian $H_{0}$.

Now we put

$$
\psi_{n}:=\left(T^{-1}\right)^{*} f_{n}, \quad n \in \mathbb{N}_{0}
$$

Then $\left\{\psi_{n}\right\}$ is a generalized Riesz system with a constructing pair $\left(\left\{f_{n}\right\},\left(T^{-1}\right)^{*}\right)$ and $\left\{\varphi_{n}\right\}$ and $\left\{\psi_{n}\right\}$ are biorthogonal sequences. For the constructing operator $\left(T^{-1}\right)^{*}$ for $\left\{\psi_{n}\right\}$, we assume the following, which is completely analogous to what stated in Assumption 1 above.

Assumption 2 Assume that there exists a dense subspace $\mathcal{E}$ in $\mathcal{H}$ satisfying

(i) $e^{-\frac{\beta}{2} H_{0}} \mathcal{H} \subset \mathcal{E}$,

(ii) $\mathcal{E} \subset D\left(T^{-1}\right) \cap D\left(\left(T^{-1}\right)^{*}\right)$,

(iii) $\left(T^{-1}\right)^{*}\lceil\mathcal{E} \in \mathcal{L}(\mathcal{E})$.

As before, we use the same symbol for the operators $\left(T^{-1}\right)^{*}, e^{-\frac{\beta}{2} H_{0}}$ and $e^{-\beta H_{0}}$ and for their restrictions to $\mathcal{E}$. Now we define put

$$
\omega_{\psi}^{\beta}(X):=\frac{1}{Z_{\psi}} \sum_{n=0}^{\infty} e^{-\beta \lambda_{n}}\left(\psi_{n} \mid \psi_{n}\right), \quad X \in \mathcal{L}^{\dagger}(\mathcal{E}),
$$

where $Z_{\psi}:=\sum_{n=0}^{\infty} e^{-\beta \lambda_{n}}\left\|\psi_{n}\right\|^{2}$, which is assumed to exist finite, see [18]. Then we have the following

Theorem 3.3 Under Assumption 2, $\omega_{\psi}^{\beta}$ is a faithful state on $\mathcal{L}^{\dagger}(\mathcal{E})$ and

$$
\begin{aligned}
\omega_{\psi}(X) & =\frac{1}{Z_{\psi}} \operatorname{tr}\left(T^{-1} X\left(T^{-1}\right)^{*} e^{-\beta H_{0}}\right) \\
& =\frac{1}{Z_{\psi}} \operatorname{tr}\left(\left(\left(T^{-1}\right)^{*} e^{-\frac{\beta}{2} H_{0}}\right)^{*} X\left(T^{-1}\right)^{*} e^{-\frac{\beta}{2} H_{0}}\right),
\end{aligned}
$$

for all $X \in \mathcal{L}^{\dagger}(\mathcal{E})$. 
Proof It is proved similarly to Theorem 3.2.

By Theorems 3.2 and 3.3 we have the following

Corollary 3.4 Let $\left\{\varphi_{n}\right\}$ and $\left\{\psi_{n}\right\}$ be biorhotogonal sequences and $\left\{\varphi_{n}\right\}$ be generalized Riesz system with natural constructing pair $\left(\left\{f_{n}\right\}, T\right)$. Suppose that Assumptions 1 and 2 are satisfied, with $\mathcal{D}=\mathcal{E}$. Then the Gibbs states $\omega_{\varphi}^{\beta}$ and $\omega_{\psi}^{\beta}$ are faithful states on $\mathcal{L}^{\dagger}(\mathcal{D})$ satisfying

$$
\begin{aligned}
\omega_{\varphi}^{\beta}(X) & =\frac{1}{Z_{\varphi}} \operatorname{tr}\left(\left(T^{*} X T\right) e^{-\beta H_{0}}\right) \\
& =\frac{1}{Z_{\varphi}} \operatorname{tr}\left(\left(T e^{-\frac{\beta}{2} H_{0}}\right)^{*} X\left(T e^{-\frac{\beta}{2} H_{0}}\right)\right)
\end{aligned}
$$

and

$$
\begin{aligned}
\omega_{\psi}^{\beta}(X) & =\frac{1}{Z_{\psi}} \operatorname{tr}\left(T^{-1} X\left(T^{-1}\right)^{*} e^{-\beta H_{0}}\right) \\
& =\frac{1}{Z_{\psi}} \operatorname{tr}\left(\left(\left(T^{-1}\right)^{*} e^{-\frac{\beta}{2} H_{0}}\right)^{*} X\left(\left(T^{-1}\right)^{*} e^{-\frac{\beta}{2} H_{0}}\right)\right),
\end{aligned}
$$

for all $X \in \mathcal{L}^{\dagger}(\mathcal{D})$.

Corollary 3.5 Let $\left\{\varphi_{n}\right\}$ be a Riesz basis with a constructing pair $\left(\left\{f_{n}\right\}, T\right)$. Suppose that there exists a dense subspace $\mathcal{D}$ in $\mathcal{H}$ such that

(i) $e^{-\frac{\beta}{2} H_{0}} \mathcal{H} \subset \mathcal{D}$.

(ii) $T \mathcal{D} \subset \mathcal{D}$.

(iii) $\left(T^{-1}\right)^{*} \mathcal{D} \subset \mathcal{D}$.

Then the Gibbs states $\omega_{\varphi}^{\beta}$ and $\omega_{\psi}^{\beta}$ are faithful states on $\mathcal{L}^{\dagger}(\mathcal{D})$.

\section{Dynamics and KMS-Like Condition}

\subsection{Standard Heisenberg Time Evolution}

Let $H_{0}$ be a non-singular positive self-adjoint operator in $\mathcal{H}$ satisfying $H_{0}=$ $\sum_{n=0}^{\infty} \lambda_{n} f_{n} \otimes f_{n}$, where $\left\{f_{n}\right\}$ is an ONB in a Hilbert space $\mathcal{H}$ and $\left\{\lambda_{n}\right\}$ is a sequence of strictly positive numbers satisfying $\sum_{n=0}^{\infty} e^{-\frac{1}{2} \lambda_{n}}<\infty$, and $\mathcal{D}$ be a dense subspace in $\mathcal{H}$ such that

$$
H_{0} \mathcal{D} \subset \mathcal{D} \text { and } e^{i t H_{0}} \mathcal{D} \subset \mathcal{D} \text { for all } t \in \mathbb{R} \text {. }
$$

For example, $\mathcal{D}=D^{\infty}\left(H_{0}\right):=\cap_{n \in \mathbb{N}_{0}} D\left(H_{0}^{n}\right)$ satisfies (4.1). Indeed, since $H_{0}^{n} e^{i t H_{0}} x=e^{i t H_{0}} H_{0}^{n} x, x \in D^{\infty}\left(H_{0}\right)$ we have $e^{i t H_{0}} x \in D\left(H_{0}^{n}\right)$, for all $n \in \mathbb{N}_{0}$. Here we put

$$
\alpha_{t}^{0}(X):=e^{i t H_{0}} X e^{-i t H_{0}}, \quad X \in \mathcal{L}^{\dagger}(\mathcal{D}), t \in \mathbb{R}
$$


Then, $\left\{\alpha_{t}^{0}\right\}_{t \in \mathbb{R}}$ is a one-parameter group of $*$-automorphisms of $\mathcal{L}^{\dagger}(\mathcal{D})$, and we have the following

Lemma 4.1.1 Suppose that (4.1) is satisfied and that the one-parameter unitary group $\left\{e^{i t H_{0}}\right\}_{t \in \mathbb{R}}$ is quasi-strongly continuous on $\mathcal{L}^{\dagger}(\mathcal{D})$.

Then, $\left\{\alpha_{t}^{0}\right\}_{t \in \mathbb{R}}$ is strongly continuous and its weak generator is $H_{0}$. In particular, if $\mathcal{D}=D^{\infty}\left(H_{0}\right)$, then $\left\{\alpha_{t}^{0}\right\}_{t \in \mathbb{R}}$ is a quasi-strongly continuous one-parameter group of *-automorphisms of $\mathcal{L}^{\dagger}\left(D^{\infty}\left(H_{0}\right)\right)$ and its quasi-strong generator is $H_{0}$.

Proof First, we show that $\left\{\alpha_{t}^{0}\right\}$ is strongly continuous. Take arbitrary $X \in \mathcal{L}^{\dagger}(\mathcal{D})$ and $\xi \in \mathcal{D}$. Then by assumption we have

$$
\begin{aligned}
\lim _{t \rightarrow 0}\left\|\alpha_{t}^{0}(X) \xi-X \xi\right\| & =\lim _{t \rightarrow 0}\left\|e^{i t H_{0}} X e^{-i t H_{0}} \xi-X \xi\right\| \\
& \leqq \lim _{t \rightarrow 0}\left\{\left\|e^{i t H_{0}} X e^{-i t H_{0}} \xi-e^{i t H_{0}} X \xi\right\|+\left\|e^{i t H_{0}} X \xi-X \xi\right\|\right\} \\
& \leqq \lim _{t \rightarrow 0}\left\|X e^{-i t H_{0}} \xi-X \xi\right\|+\lim _{t \rightarrow 0}\left\|e^{i t H_{0}} X \xi-X \xi\right\| \\
& =0 .
\end{aligned}
$$

Thus, $\left\{\alpha_{t}^{0}\right\}_{t \in \mathbb{R}}$ is strongly continuous.

Next, we show that $H_{0}$ is a weak generator of $\left\{\alpha_{t}^{0}\right\}_{t \in \mathbb{R}}$. Take arbitrary $X \in \mathcal{L}^{\dagger}(\mathcal{D})$ and $\xi, \eta \in \mathcal{D}$. Then it follows from our assumptions that

$$
\begin{aligned}
\left(\frac{\alpha_{0}^{t}(X)-X}{t} \xi \mid \eta\right) & =\left(\frac{e^{i t H_{0}} X e^{-i t H_{0}} \xi-e^{i t H_{0}} X \xi+e^{i t H_{0}} X \xi-X \xi}{t} \mid \eta\right) \\
& =\left(\frac{e^{-i t H_{0}} \xi-\xi}{t} \mid X^{\dagger} e^{-i t H_{0}} \eta\right)+\left(\frac{e^{t H_{0}} X \xi-X \xi}{t} \mid \eta\right) \\
& \rightarrow\left(-i H_{0} \xi \mid X^{\dagger} \eta\right)+\left(i H_{0} X \xi \mid \eta\right) \text { as } t \rightarrow 0 \\
& =\left(i\left[H_{0}, X\right] \xi \mid \eta\right),
\end{aligned}
$$

which yields that $H_{0}$ is a weak generator of $\left\{\alpha_{t}^{0}\right\}_{t \in \mathbb{R}}$.

Let $\mathcal{D}=D^{\infty}\left(H_{0}\right)$ and $t_{H_{0}}$ be a locally convex topology on $\mathcal{D}$ defined by a sequence $\left\{\|\cdot\|_{H_{0}^{n}} ; n \in \mathbb{N}_{0}\right\}$ of norms on $\mathcal{D}$. Since $H_{0}^{n} \in \mathcal{L}^{\dagger}(\mathcal{D})$, for all $n \in \mathbb{N}$, we have $t_{H_{0}} \prec t_{\mathcal{L}^{\dagger}(\mathcal{D})}$. Conversely we show that $t_{\mathcal{L}^{\dagger}(\mathcal{D})} \prec t_{H_{0}}$. Take an arbitrary $X \in \mathcal{L}^{\dagger}(\mathcal{D})$. Since the identity $\iota$ is a closed map of the Fréchet space $\mathcal{D}\left[t_{H_{0}}\right]$ into the Hilbert space $\mathcal{D}\left(\|\cdot\|_{\bar{X}}\right)$ with the graph norm $\|\cdot\|_{\bar{X}}:=\|\cdot\|+\|\bar{X} \cdot\|$, it follows from the closed graph theorem that it is continuous, which implies that $t_{\mathcal{L}^{\dagger}(\mathcal{D})} \prec t_{H_{0}}$. Thus we have

$$
t_{H_{0}}=t_{\mathcal{L}^{\dagger}(\mathcal{D})}
$$

and for any $X \in \mathcal{L}^{\dagger}(\mathcal{D})$ there exist $n \in \mathbb{N}$ and $\gamma>0$ such that

$$
\|X \xi\| \leqq \gamma\|\xi\|_{H_{0}^{n}} \quad \text { for all } \quad \xi \in \mathcal{D}
$$


Then, for any $X, Y \in \mathcal{L}^{\dagger}(\mathcal{D})$ and $\xi \in \mathcal{D}$ it follows from $H_{0}^{n} X \in \mathcal{L}^{\dagger}(\mathcal{D})$ and by our assumptions that

$$
\begin{aligned}
& \left\|Y \alpha_{t}^{0}(X) \xi-Y X \xi\right\| \\
& \leqq \gamma\left\|H_{0}^{n} \alpha_{t}^{0}(X) \xi-H_{0}^{n} X \xi\right\| \\
& =\gamma\left\|H_{0}^{n} e^{i t H_{0}} X e^{-i t H_{0}} \xi-H_{0}^{n} X \xi\right\| \\
& \leqq \\
& \quad \gamma\left\{\left\|H_{0}^{n} e^{i t H_{0}} X e^{-i t H_{0}} \xi-e^{i t H_{0}} H_{0}^{n} X \xi\right\|+\left\|e^{i t H_{0}} H_{0}^{n} X \xi-H_{0}^{n} X \xi\right\|\right\} \\
& =\gamma\left\{\left\|H_{0}^{n} X\left(e^{-i t H_{0}} \xi-\xi\right)\right\|+\left\|e^{i t H_{0}} H_{0}^{n} X \xi-H_{0}^{n} X \xi\right\|\right\} \\
& \quad \rightarrow 0 \text { as } t \rightarrow 0,
\end{aligned}
$$

which implies that $\alpha_{t}^{0}$ is quasi-strongly continuous. Furthermore, we have

$$
\begin{aligned}
H_{0}^{m} & \left(\frac{\alpha_{0}^{t}(X) \xi-X \xi}{t}-i\left[H_{0}, X\right] \xi\right) \\
= & H_{0}^{m}\left(\frac{e^{i t H_{0}} X e^{-i t H_{0}} \xi-e^{i t H_{0}} X \xi+e^{i t H_{0}} X \xi-X \xi}{t}-i\left[H_{0}, X\right] \xi\right) \\
= & H_{0}^{m}\left\{\left(e^{i t H_{0}} \frac{X e^{-i t H_{0}} \xi-X \xi}{t}+i X H_{0} \xi\right)+\left(\frac{e^{i t H_{0}} X \xi-X \xi}{t}-i H_{0} X \xi\right)\right\} \\
= & \left(e^{i t H_{0}} H_{0}^{m} \frac{X e^{-i t H_{0}} \xi-X \xi}{t}+i H_{0}^{m} X H_{0} \xi\right)+H_{0}^{m}\left(\frac{e^{i t H_{0}} X \xi-X \xi}{t}-i H_{0} X \xi\right) . \\
= & e^{i t H_{0}}\left(H_{0}^{m} \frac{X e^{-i t H_{0}} \xi-X \xi}{t}+i H_{0}^{m} X H_{0} \xi\right)-i H_{0}^{m}\left(e^{i t H_{0}} X H_{0} \xi-X H_{0} \xi\right) \\
& +H_{0}^{m}\left(\frac{e^{i t H_{0}} X \xi-X \xi}{t}-i H_{0} X \xi\right) .
\end{aligned}
$$

Then, it follows from $H_{0}^{m} X \in \mathcal{L}^{\dagger}(\mathcal{D})$ and (4.3) that

$$
\begin{aligned}
\left\|H_{0}^{m} X\left(\frac{e^{-i t H_{0}} \xi-\xi}{t}+i H_{0} \xi\right)\right\| \leqq & \gamma^{\prime}\left\|H_{0}^{n^{\prime}}\left(\frac{e^{-i t H_{0}} \xi-\xi}{t}+i H_{0} \xi\right)\right\| \\
= & \gamma^{\prime}\left\|\frac{e^{-i t H_{0}} H_{0}^{n^{\prime}} \xi-H_{0}^{n^{\prime}} \xi}{t}+i H_{0} H_{0}^{n^{\prime}} \xi\right\| \\
& \rightarrow \gamma^{\prime}\left\|-i H_{0} H_{0}^{n^{\prime}} \xi+i H_{0} H_{0}^{n^{\prime}} \xi\right\|=0 \text { as } t \rightarrow 0
\end{aligned}
$$

and from (ii) that

$$
H_{0}^{m}\left(e^{i t H_{0}} X H_{0} \xi-X H_{0} \xi\right) \rightarrow 0 \text { as } t \rightarrow 0
$$

which implies by (4.2) and (4.4) that $\lim _{t \rightarrow 0} \frac{\alpha_{t}^{0}(X)-X}{t}=i\left[H_{0}, X\right]$ under the quasistrong topology. This completes the proof. 


\subsection{The Heisenberg Time Evolution for Generalized Riesz Systems}

Let $\left\{\varphi_{n}\right\}$ be a generalized Riesz system with a constructing pair $\left(\left\{f_{n}\right\}, T\right)$. We assume the following

Assumption 3 There exists a dense subspace $\mathcal{D}$ in $\mathcal{H}$ such that

(i) $\left\{f_{n}\right\} \subset \mathcal{D}$, for all $n \in \mathbb{N}_{0}$,

(ii) $H_{0} \mathcal{D} \subset \mathcal{D}$ and $e^{i t H_{0}} \mathcal{D} \subset \mathcal{D}$, for all $t \in \mathbb{R}$,

(iii) $T \mathcal{D}=\mathcal{D}$ and $T^{*} \mathcal{D}=\mathcal{D}$,

(iv) $\left\{e^{i t H_{0}}\right\}_{t \in \mathbb{R}}$ is quasi-strongly continuous.

Henceforth we denote an operator $A\left\lceil_{\mathcal{D}} \in \mathcal{L}^{\dagger}(\mathcal{D})\right.$ by $A$ for simplicity. Then, we have $\varphi_{n}$, $\psi_{n}:=\left(T^{\dagger}\right)^{-1} f_{n} \in \mathcal{D}$, for all $n \in \mathbb{N}_{0}$, and we can define a non-self-adjoint operator $H$ by $H:=T H_{0} T^{-1}$. Then $H \in \mathcal{L}^{\dagger}(\mathcal{D})$ with $H^{\dagger}=\left(T^{\dagger}\right)^{-1} H_{0} T^{\dagger}$ and $H \varphi_{n}=\lambda_{n} \varphi_{n}$ and $H^{\dagger} \psi_{n}=\lambda_{n} \psi_{n}, n \in \mathbb{N}_{0}$ (we notice that (iii) implies that $\left(T^{\dagger}\right)^{-1}=\left(T^{*}\right)^{-1} \Gamma_{\mathcal{D}}$ and then $\left.\left(T^{\dagger}\right)^{-1}=\left(T^{-1}\right)^{\dagger}\right)$. Hence $H$ and $H^{\dagger}$ can be considered as non-self-adjoint hamiltonians for $\left\{\varphi_{n}\right\}$ and $\left\{\psi_{n}\right\}$, respectively. Furthermore, take arbitrary $\xi, \eta \in \mathcal{D}$ and $t \in \mathbb{R}$. By Assumption 3, (iii) there exists a element $\zeta \in \mathcal{D}$ such that $\xi=T \zeta$. Then it follows that

$$
\begin{aligned}
\left(\left(\sum_{k=0}^{n} \frac{1}{k !}(i t)^{k} H^{k}\right) \xi \mid \eta\right)= & \left(T\left(\sum_{k=0}^{n} \frac{1}{k !}(i t)^{k} H_{0}^{k}\right) T^{-1} T \zeta \mid \eta\right) \\
= & \left(\sum_{k=0}^{n} \frac{1}{k !}(i t)^{k} H_{0}^{k} \zeta \mid T^{\dagger} \eta\right) \\
& \rightarrow\left(e^{i t H_{0}} \zeta \mid T^{\dagger} \eta\right) \text { as } n \rightarrow \infty \\
= & \left(T e^{i t H_{0}} T^{-1} \xi \mid \eta\right) .
\end{aligned}
$$

Hence, $T\left(\sum_{k=0}^{n} \frac{1}{k !}(i t)^{k} H_{0}^{k}\right) T^{-1}$ converges weakly to $T e^{i t H_{0}} T^{-1}$ on $\mathcal{D}$.

Similarly, $\left(T^{\dagger}\right)^{-1}\left(\sum_{k=0}^{n} \frac{1}{k !}(i t)^{k} H_{0}^{k}\right) T^{\dagger}$ converges weakly to $\left(T^{\dagger}\right)^{-1} e^{i t H_{0}} T^{\dagger}$ on $\mathcal{D}$. Thus, it is natural to define $e^{i t H}$ and $e^{i t H^{\dagger}}$ by

$$
e^{i t H}:=T e^{i t H_{0}} T^{-1} \text { and } e^{i t H^{\dagger}}:=\left(T^{\dagger}\right)^{-1} e^{i t H_{0}} T^{\dagger} \quad t \in \mathbb{R} \text {. }
$$

Then we have the following

Lemma 4.2.1 $\left\{e^{i t H}\right\}_{t \in \mathbb{R}}$ and $\left\{e^{i t H^{\dagger}}\right\}_{t \in \mathbb{R}}$ are quasi-strongly continuous one-parameter groups of $\mathcal{L}^{\dagger}(\mathcal{D})$ satisfying $\left(e^{i t H}\right)^{\dagger}=e^{-i t H^{\dagger}}$, for all $t \in \mathbb{R}$.

Proof By (4.5) it is immediately shown that $\left\{e^{i t H}\right\}$ and $\left\{e^{i t H^{\dagger}}\right\}$ are one-parameter groups of $\mathcal{L}^{\dagger}(\mathcal{D})$ satisfying $\left(e^{i t H}\right)^{\dagger}=e^{-i t H^{\dagger}}$, for all $t \in \mathbb{R}$. We show that they are quasi-strongly continuous. Indeed, it follows from Assumption 3, (iv) that for any $X \in \mathcal{L}^{\dagger}(\mathcal{D})$ and $\xi \in \mathcal{D}$ 


$$
\begin{aligned}
\lim _{t \rightarrow 0}\left\|X e^{i t H} \xi-X \xi\right\| & =\lim _{t \rightarrow 0}\left\|X T e^{i t H_{0}} T^{-1} \xi-X \xi\right\| \\
& =\lim _{t \rightarrow 0}\left\|X T\left(e^{i t H_{0}} \eta-\eta\right)\right\| \\
& =0,
\end{aligned}
$$

where $\eta \in \mathcal{D}$ with $T \eta=\xi$.

Similarly, we can show that $\left\{e^{i t H^{\dagger}}\right\}$ is quasi-strongly continuous.

We now define what we call the Heisenberg time evolution for $\left\{\varphi_{n}\right\}$ and $\left\{\psi_{n}\right\}$ as follows:

$$
\alpha_{t}^{\varphi}(X):=e^{i t H} X e^{-i t H} \quad \text { and } \quad \alpha_{t}^{\psi}(X):=e^{i t H^{\dagger}} X e^{-i t H^{\dagger}}, \quad X \in \mathcal{L}^{\dagger}(\mathcal{D}), t \in \mathbb{R} .
$$

By (4.5) we see that

$$
\alpha_{t}^{\varphi}(X)=e^{i t H} X e^{-i t H}=T e^{i t H_{0}} T^{-1} X T e^{-i t H_{0}} T^{-1}=T \alpha_{t}^{0}\left(T^{-1} X T\right) T^{-1},
$$

where $\alpha_{t}^{0}$ was defined before. This is in complete agreement with what originally proposed in [18]. Analogously,

$$
\alpha_{t}^{\psi}(X)=\left(T^{\dagger}\right)^{-1} \alpha_{t}^{0}\left(T^{\dagger} X\left(T^{\dagger}\right)^{-1}\right) T^{\dagger}
$$

Then we have the following

Theorem 4.2.2 $\left\{\alpha_{t}^{\varphi}\right\}_{t \in \mathbb{R}}$ and $\left\{\alpha_{t}^{\psi}\right\}_{t \in \mathbb{R}}$ are weakly continuous one-parameter groups of automorphisms of $\mathcal{L}^{\dagger}(\mathcal{D})$ satisfying $\alpha_{t}^{\varphi}(X)^{\dagger}=\alpha_{t}^{\psi}\left(X^{\dagger}\right)$, for all $X \in \mathcal{L}^{\dagger}(\mathcal{D})$ and $t \in \mathbb{R}$. Furthermore their weak generators are $H$ and $H^{\dagger}$, respectively. Moreover, in particular, if $T \in B(\mathcal{H})$ (resp. $T^{-1} \in B(\mathcal{H})$ ), then $\left\{\alpha_{t}^{\varphi}\right\}$ (resp. $\left\{\alpha_{t}^{\psi}\right\}$ ) is strongly continuous.

Proof By Lemma 4.2.1, $\left\{\alpha_{t}^{\varphi}\right\}_{t \in \mathbb{R}}$ and $\left\{\alpha_{t}^{\psi}\right\}_{t \in \mathbb{R}}$ are one-parameter groups of automorphisms of $\mathcal{L}^{\dagger}(\mathcal{D})$ satisfying $\alpha_{t}^{\varphi}(X)^{\dagger}=\alpha_{t}^{\psi}\left(X^{\dagger}\right)$, for all $X \in \mathcal{L}^{\dagger}(\mathcal{D})$ and $t \in \mathbb{R}$. Let us now show that $\left\{\alpha_{t}^{\varphi}\right\}_{t \in \mathbb{R}}$ and $\left\{\alpha_{t}^{\psi}\right\}_{t \in \mathbb{R}}$ are weakly continuous. Take arbitrary $X \in \mathcal{L}^{\dagger}(\mathcal{D})$ and $\xi, \eta \in \mathcal{D}$. Since $\alpha_{t}^{\varphi}(X)=T \alpha_{t}^{0}\left(T^{-1} X T\right) T^{-1}$, for all $t \in \mathbb{R}$, it follows from Lemma 4.1.1 that

$$
\begin{aligned}
\left(\alpha_{t}^{\varphi}(X) \xi \mid \eta\right)= & \left(T \alpha_{t}^{0}\left(T^{-1} X T\right) T^{-1} T \zeta \mid \eta\right) \\
= & \left(\alpha_{t}^{0}\left(T^{-1} X T\right) \zeta \mid T^{\dagger} \eta\right) \\
& \rightarrow\left(T^{-1} X T \zeta \mid T^{\dagger} \eta\right) \text { as } t \rightarrow 0 \\
= & (X \xi \mid \eta),
\end{aligned}
$$


which yields that $\left\{\alpha_{t}^{\varphi}\right\}_{t \in \mathbb{R}}$ is weakly continuous. Next we show that $H$ is a weak generator of $\left\{\alpha_{t}^{\varphi}\right\}_{t \in \mathbb{R}}$. By Lemma 4.1.1, it follows that

$$
\begin{aligned}
\left(\left(\frac{\alpha_{t}^{\varphi}(X)-X}{t}\right) \xi \mid \eta\right)= & \left(\frac{T \alpha_{t}^{0}\left(T^{-1} X T\right) T^{-1}-X}{t} T \zeta \mid \eta\right) \\
= & \left(\frac{\alpha_{t}^{0}\left(T^{-1} X T\right)-T^{-1} X T}{t} \zeta \mid T^{\dagger} \eta\right) \\
& \rightarrow\left(i\left[H_{0}, T^{-1} X T\right] \zeta \mid T^{\dagger} \eta\right) \text { as } t \rightarrow 0 \\
= & i\left(T\left(H_{0} T^{-1} X T-T^{-1} X T H_{0}\right) \zeta \mid \eta\right) \\
= & i((H X-X H) T \zeta \mid \eta) \\
= & i([H, X] \xi \mid \eta)
\end{aligned}
$$

Thus, $H$ is a weak generator of $\left\{\alpha_{t}^{\varphi}\right\}_{t \in \mathbb{R}}$. Similarly we can show that $\left\{\alpha_{t}^{\psi}\right\}_{t \in \mathbb{R}}$ is weakly continuous and its weak generator is $H^{\dagger}$. Finally, we show that if $T \in B(\mathcal{H})$, then $\left\{\alpha_{t}^{\varphi}\right\}_{t \in \mathbb{R}}$ is strongly continuous. Take arbitrary $X \in \mathcal{L}^{\dagger}(\mathcal{D})$ and $\xi \in \mathcal{D}$. Then, as usual, there exists an element $\zeta \in \mathcal{D}$ such that $\xi=T \zeta$ and by Assumption 3, (iii) we have

$$
\begin{aligned}
\left\|\alpha_{t}^{\varphi}(X) \xi-X \xi\right\|= & \left\|T e^{i t H_{0}} T^{-1} X T e^{-i t H_{0}} T^{-1} T \zeta-T^{-1} T X T \zeta\right\| \\
\leqq & \left\|T e^{i t H_{0}} T^{-1} X T e^{-i t H_{0}} \zeta-T e^{i t H_{0}} T^{-1} X T \zeta\right\| \\
& +\left\|T e^{i t H_{0}} T^{-1} X T \zeta-T T^{-1} X T \zeta\right\| \\
\leqq & \|T\|\left\|T^{-1} X T e^{-i t H_{0}} \zeta-T^{-1} X T \zeta\right\| \\
& +\|T\|\left\|e^{i t H_{0}} T^{-1} X T \zeta-T^{-1} X T \zeta\right\| \\
& \rightarrow 0 \text { as } t \rightarrow 0 .
\end{aligned}
$$

Similarly, if $T^{-1} \in B(\mathcal{H})$, then we can show that $\left\{\alpha_{t}^{\psi}\right\}_{t \in \mathbb{R}}$ is strongly continuous. This completes the proof.

Next, let us consider the case of $\mathcal{D}=D^{\infty}\left(H_{0}\right)$. Then, Assumption 3, (i) and (ii) hold automatically, and (iv) holds from (4.1.2). Therefore, the following result easily follows.

\section{Corollary 4.2.3 Suppose that}

$$
T D^{\infty}\left(H_{0}\right)=D^{\infty}\left(H_{0}\right) \text { and } T^{*} D^{\infty}\left(H_{0}\right)=D^{\infty}\left(H_{0}\right)
$$

Then $\left\{\alpha_{t}^{\varphi}\right\}_{t \in \mathbb{R}}$ and $\left\{\alpha_{t}^{\psi}\right\}_{t \in \mathbb{R}}$ are quasi-strongly continuous and their quasi strong generators are $H$ and $H^{\dagger}$, respectively.

Proof Since $t_{\mathcal{L}^{\dagger}(\mathcal{D})}=t_{H_{0}}$ by (4.2), for any $X \in \mathcal{L}^{\dagger}(\mathcal{D})$ there exist $n \in \mathbb{N}$ and $r>0$ such that

$$
\|X \xi\| \leqq r\|\xi\|_{H_{0}^{n}} \text { for all } \xi \in \mathcal{D} \text {. }
$$


For any $X, Y \in \mathcal{L}^{\dagger}(\mathcal{D})$ and $\xi \in \mathcal{D}$ with $\xi=T \zeta$ for some $\zeta \in \mathcal{D}$, it follows from (4.6) and Assumption 3, (iv) that

$$
\begin{aligned}
\left\|Y \alpha_{t}^{\varphi}(X) \xi-Y X \xi\right\|= & \left\|Y T e^{i t H_{0}} T^{-1} X T e^{-i t H_{0}} T^{-1} T \zeta-Y T T^{-1} X T \zeta\right\| \\
\leqq & \left\|Y T\left(e^{i t H_{0}} T^{-1} X T e^{-i t H_{0}} \zeta-e^{i t H_{0}} T^{-1} X T \zeta\right)\right\| \\
& +\left\|Y T\left(e^{i t H_{0}} T^{-1} X T \zeta-T^{-1} X T \zeta\right)\right\| \\
\leqq & r_{1}\left\{\left\|H_{0}^{n_{1}} e^{i t H_{0}}\left(T^{-1} X T e^{-i t H_{0}} \eta-T^{-1} X T \eta\right)\right\|\right. \\
& \left.+\left\|H_{0}^{n_{1}}\left(e^{i t H_{0}} T^{-1} X T \eta-T^{-1} X T\right) \eta\right\|\right\} \\
= & r_{1}\left\{\left\|H_{0}^{n_{1}} T^{-1} X T\left(e^{-i t H_{0}} \zeta-\zeta\right)\right\|\right. \\
& \left.+\left\|H_{0}^{n_{1}}\left(e^{i t H_{0}} T^{-1} X T \zeta-T^{-1} X T\right) \zeta\right\|\right\} \\
\leqq & r_{1} r_{2}\left\|H_{0}^{n_{2}}\left(e^{-i t H_{0}} \zeta-\zeta\right)\right\| \\
& +r_{1}\left\|H_{0}^{n_{1}}\left(e^{i t H_{0}} T^{-1} X T \zeta-T^{-1} X T \zeta\right)\right\| \\
= & r_{1} r_{2}\left\|\left(e^{-i t H_{0}}-I\right) H_{0}^{n_{2}} \zeta\right\| \\
& +r_{1}\left\|\left(e^{i t H_{0}}-I\right)\left(H_{0}^{n_{1}} T^{-1} X T \zeta\right)\right\| \\
& \rightarrow 0 \text { as } t \rightarrow 0 .
\end{aligned}
$$

Thus $\left\{\alpha_{t}^{\varphi}\right\}$ is quasi-strongly continuous. We show that the quasi-strong generator of $\left\{\alpha_{t}^{\varphi}\right\}$ equals $H$. Indeed, take arbitrary $X, Y \in \mathcal{L}^{\dagger}(\mathcal{D})$ and $\xi \in \mathcal{D}$. Then $\xi=T \zeta$ for some $\zeta \in \mathcal{D}$ and by Lemma 4.1.1 the generator of $\left\{\alpha_{t}^{0}\right\}$ equals $H_{0}$, which yields that

$$
\begin{aligned}
\left\|Y\left(\frac{\alpha_{t}^{\varphi}(X)-X}{t}\right) \xi-Y(i[H, X]) \xi\right\| \\
=\left\|Y\left(\frac{T \alpha_{t}^{0}\left(T^{-1} X T\right) T^{-1}-X}{t}\right) T \zeta-i Y T\left[H_{0}, T^{-1} X T\right] \zeta\right\| \\
=\left\|Y T\left(\frac{\alpha_{t}^{0}\left(T^{-1} X T\right)-T^{-1} X T}{t} \zeta-i\left[H_{0}, T^{-1} X T\right] \zeta\right)\right\| \\
\quad \rightarrow 0 \text { as } t \rightarrow 0 .
\end{aligned}
$$

Thus, the quasi-strong generator of $\left\{\alpha_{t}^{\varphi}\right\}$ is $H$. Similarly, we can show that $\left\{\alpha_{t}^{\psi}\right\}$ is quasistrongly continuous and its quasi-strong generator of $\left\{\alpha_{t}^{\psi}\right\}$ is $H^{\dagger}$. This completes the proof.

\subsection{Few Words on Generalized Von Neumann Entropy}

In this section we briefly show how what is done with the dynamics can be repeated for the von Neumann entropy. We work here under a slightly generalized version of Assumption 3. In particular, we assume (i) and (iii) hold as in Assumption 3, and that $t$ in (ii) can be complex-valued, $t=t_{r}+i t_{i}$, with $t_{i}>0$. More explicitly we assume that $e^{i t H_{0}} \mathcal{D} \subset \mathcal{D}$, for all $t \in \mathbb{C}$, with $\operatorname{Im} t>0$. Assumption (3.iv) is not relevant for us here, and will not be considered. Our original assumption on the eigenvalues $\lambda_{n}$, 
$\sum_{n=0}^{\infty} e^{-\frac{1}{2} \lambda_{n}}<\infty$, is here replaced by the stronger assumptions

$$
\sum_{n=0}^{\infty} e^{-\gamma \lambda_{n}}<\infty, \quad \sum_{n=0}^{\infty} \lambda_{n} e^{-\gamma \lambda_{n}}<\infty
$$

for all $\gamma>0$. Therefore, in particular we have $Z_{0}(\beta)=\sum_{n=0}^{\infty} e^{-\beta \lambda_{n}}<\infty$. To simplify our treatment, from now on we will assume the following normalization: $Z_{0}(\beta)=1$. Here $\beta$ is just a positive parameter which, in the following section, will acquire an explicit physical meaning, the inverse temperature of a given system.

The von Neumann entropy connected to the self-adjoint Hamiltonian $H_{0}$ is defined as

$$
S_{\rho_{0}}=-\operatorname{tr}\left(\rho_{0} \log \rho_{0}\right)
$$

where, with our normalization, $\rho_{0}=e^{-\beta H_{0}}$. A straightforward computation of $S_{\rho_{0}}$ produces $S_{\rho_{0}}=\beta \sum_{n=0}^{\infty} \lambda_{n} e^{-\gamma \lambda_{n}}$, which is finite because of our assumption (4.7).

With the same steps as in the definition of $e^{i t H}$ and $e^{i t H^{\dagger}}$, using our stronger assumptions, we conclude that $\sum_{k=0}^{n} \frac{1}{k !}(-\beta)^{k} H^{k}$ converges weakly to $T e^{-\beta H_{0}} T^{-1}=$ $T \rho_{0} T^{-1}$ on $\mathcal{D}$, and $\sum_{k=0}^{n} \frac{1}{k !}(-\beta)^{k} H^{\dagger^{k}}$ converges weakly to $\left(T^{\dagger}\right)^{-1} e^{-\beta H_{0}} T^{\dagger}=$ $\left(T^{\dagger}\right)^{-1} \rho_{0} T^{\dagger}$ on $\mathcal{D}$. This suggests to define, in analogy with (4.5),

$$
\rho=T \rho_{0} T^{-1}, \quad \rho^{\dagger}=\left(T^{\dagger}\right)^{-1} \rho_{0} T^{\dagger} .
$$

Notice now that $(\rho-11)^{k}=T\left(\rho_{0}-11\right)^{k} T^{-1}$, for all $k=0,1,2, \ldots$ Therefore, using the same argument bringing to definitions (4.5), we can check that $\sum_{k=1}^{n}(-1)^{k-1} \frac{1}{k}(\rho-$ $11)^{k}$ converges weakly to $T \log \left(\rho_{0}\right) T^{-1}$ on $\mathcal{D}$, and $\sum_{k=0}^{n}(-1)^{k-1} \frac{1}{k}\left(\rho^{\dagger}-11\right)^{k}$ converges weakly to $\left(T^{\dagger}\right)^{-1} \log \left(\rho_{0}\right) T^{\dagger}$ on $\mathcal{D}$. Hence we put

$$
\log \rho:=T \log \left(\rho_{0}\right) T^{-1}, \quad \log \rho^{\dagger}:=\left(T^{\dagger}\right)^{-1} \log \left(\rho_{0}\right) T^{\dagger},
$$

and we define a new von Neumann-like entropy as follows:

$$
S_{\rho}=-\sum_{n}\left(\psi_{n} \mid \rho \log \rho \varphi_{n}\right)=-\sum_{n}\left(\rho^{\dagger} \psi_{n} \mid(\log \rho) \varphi_{n}\right)
$$

under our working assumptions, and in particular the fact that $\rho^{\dagger} \psi_{n} \in \mathcal{D}$ and $(\log \rho) \varphi_{n} \in \mathcal{D}$, we easily conclude that $S_{\rho}=S_{\rho_{0}}$.

Remark It is worth pointing out that even in the cases when $H_{0} \mathcal{D} \subset \mathcal{D}$, we cannot say that $\log \left(\rho_{0}\right) \in \mathcal{L}^{\dagger}(D)$ as it happens if $\mathcal{D}=D^{\infty}\left(H_{0}\right)$; due to the assumptions on $T$ $\left(T \mathcal{D}=\mathcal{D} ; T^{*} D=D\right)$ this would imply that also $\log (\rho)$ maps $\mathcal{D}$ into $\mathcal{D}$. Nevertheless in the above computations only the action the $\left\{\varphi_{n}\right\}$ 's is involved, were everything goes in the appropriate way. 


\subsection{KMS-Like Condition}

In this section we investigate whether the Gibbs state $\omega_{\varphi}^{\beta}$ satisfies the KMS-condition with respect to $\left\{\alpha_{t}^{\varphi}\right\}$, that is, for any $X, Y \in \mathcal{L}^{\dagger}(\mathcal{D})$ there exists a bounded continuous function $f_{X, Y}$ on the $\operatorname{strip} \mathcal{S}_{\beta}:=\{z \in \mathbb{C} ; 0 \leqq \operatorname{Im} z \leqq \beta\}$ such that

$$
\begin{aligned}
f_{X, Y}(t) & =\omega_{\varphi}^{\beta}\left(X \alpha_{t}^{\varphi}(Y)\right), \\
f_{X, Y}(t+\beta i) & =\omega_{\varphi}^{\beta}\left(\alpha_{t}^{\varphi}(Y) X\right),
\end{aligned}
$$

for all $t \in \mathbb{R}$.

Throughout this section let $\left\{\varphi_{n}\right\}$ be a generalized Riesz system in $\mathcal{H}$ with a constructing pair $\left(\left\{f_{n}\right\}, T\right)$ satisfying $T D^{\infty}\left(H_{0}\right)=D^{\infty}\left(H_{0}\right)$ and $T^{*} D^{\infty}\left(H_{0}\right)=D^{\infty}\left(H_{0}\right)$ and $\beta>0$. Here we put $\mathcal{D}:=D^{\infty}\left(H_{0}\right)$. Then, since $e^{-\delta H_{0}} \mathcal{H} \subset \mathcal{D}$ for any $\delta>0$, it follows from Lemma 3.1 that

$$
X e^{-\delta H_{0}} \text { is trace class for each } \delta>0 \text { and } X \in \mathcal{L}^{\dagger}(\mathcal{D}, \mathcal{H}),
$$

and from Corollarys 3.4 and 4.2.3 that $\omega_{\varphi}^{\beta}$ and $\omega_{\psi}^{\beta}$ are faithful states on $\mathcal{L}^{\dagger}(\mathcal{D})$ and $\left\{\alpha_{t}^{\varphi}\right\}_{t \in \mathbb{R}}$ and $\left\{\alpha_{t}^{\psi}\right\}_{t \in \mathbb{R}}$ are quasi-strongly continuous one-parameter groups of automorphisms of $\mathcal{L}^{\dagger}(\mathcal{D})$. We have the following

Theorem 4.4.1 For any $X, Y \in \mathcal{L}^{\dagger}(\mathcal{D})$ there exists a bounded continuous function $f_{X, Y}$ on the strip $\mathcal{S}_{\beta}$ in $\mathbb{C}$ which is analytic on $0<\operatorname{Im} z<\beta$ such that

$$
\begin{aligned}
f_{X, Y}(t) & =\omega_{\varphi}^{\beta}\left(X \alpha_{t}^{\varphi}(Y)\right), \\
f_{X, Y}(t+\beta i) & =\omega_{\varphi}^{\beta}\left(\left(T T^{\dagger}\right)^{-1} \alpha_{t}^{\varphi}(Y) T T^{\dagger} X\right),
\end{aligned}
$$

for all $t \in \mathbb{R}$.

Proof By Theorem 3.2 we have

$$
\begin{aligned}
\omega_{\varphi}^{\beta}(X) & =\frac{1}{Z_{\varphi}} \operatorname{tr}\left(T^{\dagger} X T e^{-\beta H_{0}}\right) \\
& =\frac{1}{Z_{\varphi}} \operatorname{tr}\left(T e^{-\beta H_{0}} T^{\dagger} X\right) \\
& =\frac{1}{Z_{\varphi}} \operatorname{tr}\left(e^{-\beta H} T T^{\dagger} X\right),
\end{aligned}
$$

for all $X \in \mathcal{L}^{\dagger}(\mathcal{D})$. In order to define a function $f_{X, Y}$ on the strip $\mathcal{S}_{\beta}$ in $\mathbb{C}$, we extend $\alpha_{t}^{\varphi}$ to the $\operatorname{strip} \mathcal{S}_{\beta}$ as follows:

$$
\begin{aligned}
\alpha_{z}^{\varphi}(Y) & =T e^{i z H_{0}} T^{-1} Y T e^{-i z H_{0}} T^{-1} \\
& =T e^{-s H_{0}} e^{i t H_{0}} T^{-1} Y T e^{-i t H_{0}} e^{s H_{0}} T^{-1}, \quad z=t+i s \in \mathcal{S}_{\beta} \text { and } Y \in \mathcal{L}^{\dagger}(\mathcal{D})
\end{aligned}
$$


Then, $\alpha_{z}^{\varphi}(Y)$ is not necessarily contained in $\mathcal{L}^{\dagger}(\mathcal{D})$. However, we have

$$
\begin{aligned}
T^{\dagger} X \alpha_{z}^{\varphi}(Y) T e^{-\beta H_{0}} & =T^{\dagger} X T e^{-s H_{0}} e^{i t H_{0}} T^{-1} Y T e^{-i t H_{0}} e^{s H_{0}} e^{-\beta H_{0}} \\
& =T^{\dagger} X T e^{-s H_{0}} \alpha_{t}^{0}\left(T^{-1} Y T\right) e^{-(\beta-s) H_{0}}
\end{aligned}
$$

Hence, because of (4.8), taking into account that $\left.T^{\dagger} X T e^{-s H_{0}} \alpha_{t}^{0}\left(T^{-1} Y T\right) \in \mathcal{L}^{\dagger}(\mathcal{D})\right)$, we conclude that $T^{\dagger} X \alpha_{z}^{\varphi}(Y) T e^{-\beta H_{0}}$ is trace class. Now we put

$$
f_{X, Y}(z):=\frac{1}{Z_{\varphi}} \operatorname{tr}\left(T^{\dagger} X \alpha_{z}^{\varphi}(Y) T e^{-\beta H_{0}}\right), \quad z \in \mathcal{S}_{\beta}
$$

Then $f_{X, Y}(z)$ is analytic on $z \in \mathcal{S}_{\beta}$ with $0<\operatorname{Im} z<\beta$. Indeed, take arbitrary a sufficient small constant $\delta>0(0<\delta<\beta)$. Then we have

$$
\begin{aligned}
f_{X, Y}(z) & =\frac{1}{Z_{\varphi}} \operatorname{tr}\left(T^{\dagger} X T e^{i z H_{0}} T^{-1} Y T e^{-i z H_{0}} T^{-1} T e^{-\beta H_{0}}\right) \\
& =\frac{1}{Z_{\varphi}} \operatorname{tr}\left(T^{\dagger} X T e^{i z H_{0}} T^{-1} Y T e^{-i z H_{0}} T^{-1} T e^{-(\beta-\delta) H_{0}} e^{-\delta H_{0}}\right) \\
& =\frac{1}{Z_{\varphi}} \operatorname{tr}\left(e^{-(\beta-\delta) H_{0}}\left(e^{-\frac{\delta}{2} H_{0}} T^{\dagger} X T\right) e^{i z H_{0}}\left(T^{-1} Y T e^{-\frac{\delta}{2} H_{0}}\right) e^{-i z H_{0}}\right) \\
& =\frac{1}{Z_{\varphi}} \operatorname{tr}\left(e^{-(\beta-\delta) H_{0}}\left(e^{-\frac{\delta}{2} H_{0}} T^{-1} X T\right) \alpha_{z}^{0}\left(T^{-1} Y T e^{-\frac{\delta}{2} H_{0}}\right)\right) \\
& =\frac{1}{Z_{\varphi}} \operatorname{tr}\left(e^{-(\beta-\delta) H_{0}} A \alpha_{z}^{0}(B)\right)
\end{aligned}
$$

where $A:=\left(e^{-\frac{\delta}{2} H_{0}} T^{-1} X T\right)$ and $B:=\left(T^{-1} Y T e^{-\frac{\delta}{2} H_{0}}\right)$. By (4.8), $A$ and $B$ are trace class. Hence it is known that

$$
z \rightarrow \operatorname{tr}\left(e^{-(\beta-\delta) H_{0}} A \alpha_{0}^{z}(B)\right) \text { is analytic on } \mathcal{S}_{\beta-\delta} \text { with } 0<\operatorname{Im} z<\beta-\delta
$$

(see 4.3 in [24]). Then for any $z_{0} \in \mathcal{S}_{\beta}$ with $0<\operatorname{Im} z_{0}<\beta$ there exists a constant $\delta>0$ such that $\operatorname{Im} z_{0}<\beta-\delta$. By (4.11), $f_{X, Y}$ is analytic at $z_{0}$. Thus $f_{X, Y}$ is analytic on $\mathcal{S}_{\beta}$ with $0<\operatorname{Im} z<\beta$. Furthermore, by (4.9) we have

$$
\begin{aligned}
f_{X, Y}(t) & =\frac{1}{Z_{\varphi}} \operatorname{tr}\left(T^{\dagger} X \alpha_{t}^{\varphi}(Y) T e^{-\beta H_{0}}\right) \\
& =\frac{1}{Z_{\varphi}} \operatorname{tr}\left(T e^{-\beta H_{0}} T^{\dagger} X \alpha_{t}^{\varphi}(Y)\right) \\
& =\omega_{\varphi}^{\beta}\left(X \alpha_{t}^{\varphi}(Y)\right)
\end{aligned}
$$


and

$$
\begin{aligned}
& f_{X, Y}(t+\beta i) \\
&=\frac{1}{Z_{\varphi}} \operatorname{tr}\left(T e^{-\beta H_{0}} T^{-1} T T^{\dagger} X\left(T e^{-\beta H_{0}} T^{-1}\right)\right. \\
&\left.\left(T e^{i t H_{0}}\right) T^{-1} Y\left(T e^{-i t H_{0}} T^{-1}\right)\left(T e^{-\beta H_{0}} T^{-1}\right)\right) \\
&= \frac{1}{Z_{\varphi}} \operatorname{tr}\left(T T^{\dagger} X\left(T e^{-\beta H_{0}} T^{-1}\right) T \alpha_{t}^{0}\left(T^{-1} Y T\right) T^{-1}\right) \\
&= \frac{1}{Z_{\varphi}} \operatorname{tr}\left(e^{-\beta H} T \alpha_{t}^{0}\left(T^{-1} Y T\right) T^{\dagger} X\right) \\
&= \frac{1}{Z_{\varphi}} \operatorname{tr}\left(e^{-\beta H} \alpha_{t}^{\varphi}(Y) T T^{\dagger} X\right) \\
&= \frac{1}{Z_{\varphi}} \operatorname{tr}\left(e^{-\beta H} T T^{\dagger}\left(T T^{\dagger}\right)^{-1} \alpha_{t}^{\varphi}(Y) T T^{\dagger} X\right) \\
&= \omega_{\varphi}^{\beta}\left(\left(T T^{\dagger}\right)^{-1} \alpha_{t}^{\varphi}(Y) T T^{\dagger} X\right) .
\end{aligned}
$$

This completes the proof.

Thus $\omega_{\varphi}^{\beta}$ does not satisfy the KMS-condition with respect to $\left\{\alpha_{t}^{\varphi}\right\}$, but still it satisfies the KMS-like condition with respect to $\left\{\alpha_{t}^{\varphi}\right\}$, as Theorem 4.4.1 shows. Furthermore, we have a similar result for the Gibbs state $\omega_{\psi}^{\beta}$ as follows:

Theorem 4.4.2 For any $X, Y \in \mathcal{L}^{\dagger}(\mathcal{D})$ there exists a bounded continuous function $F_{X, Y}$ on the strip $\mathcal{S}_{\beta}$ in $\mathbb{C}$ which is analytic on $0<\operatorname{Im} z<\beta$ such that

$$
\begin{aligned}
F_{X, Y}(t) & =\omega_{\psi}^{\beta}\left(X \alpha_{t}^{\psi}(Y)\right), \\
F_{X, Y}(t+\beta i) & =\omega_{\psi}^{\beta}\left(\left(T T^{\dagger}\right) \alpha_{t}^{\psi}(Y)\left(T T^{\dagger}\right)^{-1} X\right),
\end{aligned}
$$

for all $t \in \mathbb{R}$.

Remark We do not know whether Theorems 4.4.1 and 4.4.2 hold for a general subspace $\mathcal{D}$ satisfying Assumption 3. This is because we do not know whether (4.10) holds for unbounded operators $T^{\dagger} X \alpha_{z}^{\varphi}(Y) T$.

\section{Gibbs States and Unbounded Tomita-Takesaki Theory}

\subsection{Unbounded Tomita-Takesaki Theory in Hilbert Space of Hilbert-Schmidt Operators}

In this subsection we review the basic definitions and results of unbounded TomitaTakesaki theory in the Hilbert space of Hilbert-Schmidt operators. For details refer 
to [25]. Let $\mathcal{H}$ be a separable Hilbert space and $\mathcal{H} \otimes \overline{\mathcal{H}}$ be the Hilbert space of all Hilbert-Schmidt operators on $\mathcal{H}$ with the inner product

$$
(S \mid T):=\operatorname{tr}\left(T^{*} S\right), \quad S, T \in \mathcal{H} \otimes \overline{\mathcal{H}} .
$$

Let $\mathcal{D}$ be a dense subspace in $\mathcal{H}$ such that $\mathcal{L}^{\dagger}(\mathcal{D})$ is closed, namely $\mathcal{D}=$ $\cap_{X \in \mathcal{L}^{\dagger}(\mathcal{D})} D(\bar{X})$. We define a dense subspace $\sigma_{2}(\mathcal{D})$ of $\mathcal{H} \otimes \overline{\mathcal{H}}$ by

$$
\sigma_{2}(\mathcal{D}):=\left\{T \in \mathcal{H} \otimes \overline{\mathcal{H}} ; T \mathcal{H} \subset \mathcal{D} \text { and } X T \in \mathcal{H} \otimes \overline{\mathcal{H}} \text { for all } X \in \mathcal{L}^{\dagger}(\mathcal{D})\right\}
$$

and an operator $\pi(X)$ on $\sigma_{2}(\mathcal{D})$ by

$$
\pi(X):=X T, \quad X \in \mathcal{L}^{\dagger}(\mathcal{D}), T \in \sigma_{2}(\mathcal{D})
$$

Then $\pi$ is a $*$-homomorphism of the $O^{*}$-algebra $\mathcal{L}^{\dagger}(\mathcal{D})$ into the $O^{*}$-algebra $\mathcal{L}^{\dagger}\left(\sigma_{2}(\mathcal{D})\right)$, and hence $\pi\left(\mathcal{L}^{\dagger}(\mathcal{D})\right)$ is an $O^{*}$-algebra on $\sigma_{2}(\mathcal{D})$ in $\mathcal{H} \otimes \overline{\mathcal{H}}$. We can also define a bounded $*$-homomorphism $\pi^{\prime \prime}$ and an anti $*$-homomorphism $\pi^{\prime}$ of $B(\mathcal{H})$ into the $C^{*}$-algebra $B(\mathcal{H} \otimes \overline{\mathcal{H}})$ by

$$
\pi^{\prime \prime}(A) T=A T \text { and } \pi^{\prime}(A) T=T A, \quad A \in B(\mathcal{H}), T \in \mathcal{H} \otimes \overline{\mathcal{H}}
$$

and $\pi^{\prime \prime}(B(\mathcal{H}))$ and $\pi^{\prime}(B(\mathcal{H}))$ are von Neumann algebras on $\mathcal{H} \otimes \overline{\mathcal{H}}$ satisfying $\pi^{\prime}(B(\mathcal{H}))=\pi^{\prime \prime}(B(\mathcal{H}))^{\prime}=J \pi^{\prime \prime}(B(\mathcal{H})) J$, where $J T=T^{*}$ for any $T \in \mathcal{H} \otimes \overline{\mathcal{H}}$. Then it follows from Lemma 2.4.14 in [25] that

$$
\pi\left(\mathcal{L}^{\dagger}(\mathcal{D})\right)_{w}^{\prime}=\pi^{\prime}(B(\mathcal{H})) \text { and }\left(\pi\left(\mathcal{L}^{\dagger}(\mathcal{D})\right)_{w}^{\prime}\right)^{\prime}=\pi^{\prime \prime}(B(\mathcal{H}))
$$

Suppose that $\Omega$ is a non-singular positive self-adjoint operator on $\mathcal{H}$ belonging to $\sigma_{2}(\mathcal{D})$. Then, it follows from Lemma 2.4.16 in [25] that $\Omega$ is a strongly cyclic vector for the $O^{*}$-algebra $\pi\left(\mathcal{L}^{\dagger}(\mathcal{D})\right.$ ) (namely, $\pi\left(\mathcal{L}^{\dagger}(\mathcal{D})\right) \Omega$ is $t_{\pi\left(\mathcal{L}^{\dagger}(\mathcal{D}) \text { ) }\right.}$-dense in $\mathcal{H} \otimes \overline{\mathcal{H}}$ ) and $\pi\left(\mathcal{L}^{\dagger}(\mathcal{D})\right)_{w}^{\prime} \Omega$ is dense in $\mathcal{H} \otimes \overline{\mathcal{H}}$, and hence it is a cyclic and separating vector for the von Neumann algebra $\pi^{\prime \prime}(B(\mathcal{H}))$, which implies that $\pi^{\prime \prime}(B(\mathcal{H})) \Omega$ is a left Hilbert algebra in $\mathcal{H} \otimes \overline{\mathcal{H}}$ under the following multiplication and involution:

$$
\begin{aligned}
\left(\pi^{\prime \prime}(A) \Omega\right)\left(\pi^{\prime \prime}(B) \Omega\right) & :=\pi^{\prime \prime}(A B) \Omega, \\
\left(\pi^{\prime \prime}(A) \Omega\right)^{\sharp} & :=\pi^{\prime \prime}\left(A^{*}\right) \Omega, \quad A, B \in B(\mathcal{H}) .
\end{aligned}
$$

Let $S_{\mathfrak{A}}^{\prime \prime}=J_{\Omega}^{\prime \prime} \triangle_{\Omega}^{\prime \prime \frac{1}{2}}$ be the polar decomposition of the conjugate linear closed operator $S_{\Omega}^{\prime \prime}$ which is the closure of the involution $\pi^{\prime \prime}(A) \Omega \rightarrow \pi^{\prime \prime}\left(A^{*}\right) \Omega$. Then $J_{\Omega}^{\prime \prime}$ is a conjugate linear isometry on $\mathcal{H} \otimes \overline{\mathcal{H}}$ and $\triangle_{\Omega}^{\prime \prime}$ is a non-singular positive self-adjoint operator in $\mathcal{H} \otimes \overline{\mathcal{H}}$ and they are called the modular conjugation and the modular operator of the left Hilbert algebra $\pi^{\prime \prime}(B(\mathcal{H})) \Omega$. By the Tomita theorem a strongly continuous 
one-parameter group $\left\{\left(\delta_{t}^{\Omega}\right)^{\prime \prime}\right\}_{t \in \mathbb{R}}$ of the von Neumann algebra $\pi^{\prime \prime}(B(\mathcal{H}))$ is defined by

$$
\left(\delta_{t}^{\Omega}\right)^{\prime \prime}\left(\pi^{\prime \prime}(A)\right)=\triangle_{\Omega}^{\prime \prime}{ }^{i t} \pi^{\prime \prime}(A) \triangle_{\Omega}^{\prime \prime}-i t, \quad A \in B(\mathcal{H}), t \in \mathbb{R}
$$

and it is called the modular automorphism group of $\pi^{\prime \prime}(B(\mathcal{H}))$. For the TomitaTakesaki theory we refer to [26]. Then it follows from Theorem 2.4.18 in [25] that

$$
J_{\Omega}^{\prime \prime}=J \text { and } \triangle_{\Omega}^{\prime \prime}=\pi^{\prime}\left(\Omega^{-2}\right) \pi^{\prime \prime}\left(\Omega^{2}\right)
$$

where the positive self-adjoint operator $\pi^{\prime}\left(\Omega^{-2}\right)$ is defined by

$$
\left\{\begin{aligned}
D\left(\pi^{\prime}\left(\Omega^{-2}\right)\right)= & \left\{T \in \mathcal{H} \otimes \overline{\mathcal{H}} ; T \Omega^{-2} \text { is closable and } \overline{T \Omega^{-2}} \in \mathcal{H} \otimes \overline{\mathcal{H}}\right\} \\
& \pi^{\prime}\left(\Omega^{-2}\right) T=\overline{T \Omega^{-2}}, T \in D\left(\pi^{\prime}\left(\Omega^{-2}\right)\right) .
\end{aligned}\right.
$$

By (5.1) we have

$$
\begin{aligned}
\pi\left(\mathcal{L}^{\dagger}(\mathcal{D})\right)_{w}^{\prime} \Omega & =\pi^{\prime}(B(\mathcal{H})) \Omega, \\
\left(\pi\left(\mathcal{L}^{\dagger}(\mathcal{D})\right)_{w}^{\prime}\right)^{\prime} \Omega & =\pi^{\prime \prime}(B(\mathcal{H})) \Omega,
\end{aligned}
$$

and so the involution: $\pi(X) \Omega \rightarrow \pi\left(X^{\dagger}\right) \Omega, X \in \mathcal{L}^{\dagger}(\mathcal{D})$ is a conjugate linear closable operator in $\mathcal{H} \otimes \overline{\mathcal{H}}$ and its closure is denoted by $S_{\mathfrak{A}}$. Let $S_{\mathfrak{A}}=J_{\mathfrak{A}} \triangle_{\mathfrak{A}}^{\frac{1}{2}}$ be the polar decomposition of $S_{\mathfrak{A}}$. Then we can show that $S_{\mathfrak{A}}=S_{\mathfrak{A}}^{\prime \prime}$, and so $J_{\mathfrak{A}}=J_{\mathfrak{A}}^{\prime \prime}$ and $\triangle_{\mathfrak{A}}=\triangle_{\mathfrak{A}}^{\prime \prime}$. Hereafter, we use $S_{\mathfrak{A}}, J_{\mathfrak{A}}, \triangle_{\mathfrak{A}}$ and $\left\{\delta_{t}^{\Omega}\right\}_{t \in \mathbb{R}}$. Suppose that $\Omega^{i t} \mathcal{D} \subset \mathcal{D}$, for all $t \in \mathbb{R}$, namely $\Omega^{i t} \in \mathcal{L}^{\dagger}(\mathcal{D})$. Then since

$$
\triangle_{\Omega}^{i t}=\pi^{\prime}\left(\Omega^{-2 i t}\right) \pi^{\prime \prime}\left(\Omega^{2 i t}\right)=\pi^{\prime}\left(\Omega^{-2 i t}\right) \pi\left(\Omega^{2 i t}\right) \in \mathcal{L}^{\dagger}(\mathcal{D}), \quad t \in \mathbb{R}
$$

by (5.2), we can define a one-parameter group $\left\{\sigma_{t}^{\Omega}\right\}_{t \in \mathbb{R}}$ of the $O^{*}$-algebra $\pi\left(\mathcal{L}^{\dagger}(\mathcal{D})\right)$ by

$$
\sigma_{t}^{\Omega}(\pi(X)):=\triangle_{\Omega}^{i t} \pi(X) \triangle_{\Omega}^{-i t}, \quad X \in \mathcal{L}^{\dagger}(\mathcal{D}), t \in \mathbb{R}
$$

and we see that

$$
\begin{aligned}
\sigma_{t}^{\Omega}(\pi(X)) & =\pi^{\prime}\left(\Omega^{-2 i t}\right) \pi\left(\Omega^{2 i t}\right) \pi(X) \pi^{\prime}\left(\Omega^{2 i t}\right) \pi\left(\Omega^{-2 i t}\right) \\
& =\pi\left(\Omega^{2 i t}\right) \pi(X) \pi\left(\Omega^{-2 i t}\right) \\
& =\pi\left(\Omega^{2 i t} X \Omega^{-2 i t}\right)
\end{aligned}
$$

for all $X \in \mathcal{L}^{\dagger}(\mathcal{D})$ and $t \in \mathbb{R}$. This $\left\{\sigma_{t}^{\Omega}\right\}$ is called the modular automorphism group of $\pi\left(\mathcal{L}^{\dagger}(\mathcal{D})\right)$. Thus we have the following 
Proposition 5.1.1 Suppose that $\Omega$ is a non-singular positive self-adjoint operator on $\mathcal{H}$ belonging to $\sigma_{2}(\mathcal{D})$ and $\Omega^{i t} \mathcal{D} \subset \mathcal{D}$, for all $t \in \mathbb{R}$. Then

$$
\sigma_{t}^{\Omega}(X):=\Omega^{i t} X \Omega^{-i t}, \quad X \in \mathcal{L}^{\dagger}(\mathcal{D}), t \in \mathbb{R}
$$

is a one-parameter group of $*$-automorphisms of $\mathcal{L}^{\dagger}(\mathcal{D})$, which is induced by the modular automorphism group $\left\{\sigma_{t}^{\Omega}\right\}_{t \in \mathbb{R}}$ of $\pi\left(\mathcal{L}^{\dagger}(\mathcal{D})\right)$.

\subsection{Modular Automorphism Group Defined by the Gibbs State $\omega_{\varphi}^{\beta}$}

Let $\left\{\varphi_{n}\right\}$ be a generalized Riesz system with a constructing pair $\left(\left\{f_{n}\right\}, T\right)$ and $H_{0}$ be a standard Hamiltonian. We assume the following

Assumption 4 There exists a dense subspace $\mathcal{D}$ in $\mathcal{H}$ such that

(i) $e^{-\frac{\beta}{2} H_{0}} \mathcal{H} \subset \mathcal{D} \subset D(T) \cap D\left(T^{*}\right)$,

(ii) $T\lceil\mathcal{D} \in \mathcal{L}(\mathcal{D})$,

(iii) $\mathcal{L}^{\dagger}(\mathcal{D})$ is self-adjoint, namely $\mathcal{D}=\cap_{X \in \mathcal{L}^{\dagger}(\mathcal{D})} D\left(X^{*}\right)$.

As seen in Sect. 3, the Gibbs state $\omega_{0}$ on $\mathcal{L}^{\dagger}(\mathcal{D})$ is defined by

$$
\omega_{0}(X)=\frac{1}{Z_{0}} \operatorname{tr}\left(e^{-\frac{\beta}{2} H_{0}} X e^{-\frac{\beta}{2} H_{0}}\right), \quad X \in \mathcal{L}^{\dagger}(\mathcal{D}) .
$$

Then we see that

$$
\Omega_{0}:=\frac{1}{\sqrt{Z_{0}}} e^{-\frac{\beta}{2} H_{0}} \in\left(\sigma_{2}\left(\mathcal{L}^{\dagger}(\mathcal{D})\right)\right)_{+}
$$

and

$$
\omega_{0}(X)=\left(\pi(X) \Omega_{0} \mid \Omega_{0}\right), \quad X \in \mathcal{L}^{\dagger}(\mathcal{D})
$$

By Proposition 5.1.1, the modular automorphism group $\left\{\sigma_{t}^{\Omega_{0}}\right\}_{t \in \mathbb{R}}$ of $\pi\left(\mathcal{L}^{\dagger}(\mathcal{D})\right)$ defined by $\omega_{0}$ coincides with $\left\{\alpha_{t}^{0}\right\}_{t \in \mathbb{R}}$. By Theorem 3.2, the Gibbs state $\omega_{\varphi}^{\beta}$ for $\left\{\varphi_{n}\right\}$ is defined by

$$
\omega_{\varphi}^{\beta}(X)=\frac{1}{Z_{\varphi}} \operatorname{tr}\left(\left(T e^{-\frac{\beta}{2} H_{0}}\right)^{*} X\left(T e^{-\frac{\beta}{2} H_{0}}\right)\right),
$$

for all $X \in \mathcal{L}^{\dagger}(\mathcal{D})$. Here we shall extend results in Sect. 3 for the Gibbs state $\omega_{\varphi}^{\beta}$ on $\mathcal{L}^{\dagger}(\mathcal{D})$.

Let $\left(T e^{-\frac{\beta}{2} H_{0}}\right)^{*}=U\left|\left(T e^{-\frac{\beta}{2} H_{0}}\right)^{*}\right|$ be the polar decomposition of $\left(T e^{-\frac{\beta}{2} H_{0}}\right)^{*}$. By Lemma 3.1 and Assumption 4, (i) and (ii), we have

$$
\left(T e^{-\frac{\beta}{2} H_{0}}\right)\left(T e^{-\frac{\beta}{2} H_{0}}\right)^{*} \mathcal{H} \subset T\left(e^{-\frac{\beta}{2} H_{0}}\left(T e^{-\frac{\beta}{2} H_{0}}\right)^{*}\right) \mathcal{H} \subset T \mathcal{D} \subset \mathcal{D}
$$


and since $\mathcal{L}^{\dagger}(\mathcal{D})$ is a self-adjoint $O^{*}$-algebra on $\mathcal{D}$, it follows by Lemma 2.4 in [27] that

$$
\left|\left(T e^{-\frac{\beta}{2} H_{0}}\right)^{*}\right| \mathcal{H}=\left(\left(T e^{-\frac{\beta}{2} H_{0}}\right)\left(T e^{-\frac{\beta}{2} H_{0}}\right)^{*}\right)^{\frac{1}{2}} \mathcal{H} \subset \mathcal{D} .
$$

From the above, we put

$$
\Omega_{\varphi}:=\frac{1}{\sqrt{Z_{\varphi}}}\left|\left(T e^{-\frac{\beta}{2} H_{0}}\right)^{*}\right|
$$

Since $X T \in \mathcal{L}^{\dagger}(\mathcal{D}, \mathcal{H})$ for all $X \in \mathcal{L}^{\dagger}(\mathcal{D})$ by Assumption 4, (i), it follows from Lemma 3.1 that

$$
\begin{aligned}
X \Omega_{\varphi} & =\frac{1}{\sqrt{Z_{\varphi}}} X\left|\left(T e^{-\frac{\beta}{2} H_{0}}\right)^{*}\right| \\
& =\frac{1}{\sqrt{Z_{\varphi}}} X T e^{-\frac{\beta}{2} H_{0}} U \in \mathcal{H} \otimes \overline{\mathcal{H}}
\end{aligned}
$$

for all $X \in \mathcal{L}^{\dagger}(\mathcal{D})$, which implies by (5.3) that $\Omega_{\varphi} \in \sigma_{2}(\mathcal{D})$. Thus, $\Omega_{\varphi}$ is a non-singular positive self-adjoint Hilbert Schmidt operator on $\mathcal{H}$ contained in $\sigma_{2}(\mathcal{D})$. Hence, $\Omega_{\varphi}$ is a strongly cyclic and separating vector for $\pi\left(\mathcal{L}^{\dagger}(\mathcal{D})\right)$ and

$$
\begin{aligned}
\omega_{\varphi}^{\beta}(X) & =\operatorname{tr}\left(U \Omega_{\varphi} X \Omega_{\varphi} U^{*}\right) \\
& =\operatorname{tr}\left(\Omega_{\varphi} X \Omega_{\varphi}\right) \\
& =\left(\pi(X) \Omega_{\varphi} \mid \Omega_{\varphi}\right), \quad X \in \mathcal{L}^{\dagger}(\mathcal{D})
\end{aligned}
$$

Remark The previous expression for $\omega_{\varphi}$ is, of course, the GNS representation (up to unitary equivalences) and the cyclic and separating vector $\Omega_{\varphi}$ which is actually an operator in $\sigma_{2}(\mathcal{D})$ helps with identifying the density operator $\rho$ for which one can write $\omega_{\varphi}^{\beta}(X)=\operatorname{tr}(X \rho)$.

By Proposition 5.1.1, we have the following

Theorem 5.2.1 Suppose that $\left\{\varphi_{n}\right\}$ be a generalized Riesz system with a constructing pair $\left(\left\{f_{n}\right\}, T\right)$ and there exists a dense subspace $\mathcal{D}$ in $\mathcal{H}$ satisfying Assumption 4 . Then $\Omega_{\varphi}:=\frac{1}{\sqrt{Z_{\varphi}}}\left|\left(T e^{-\frac{\beta}{2} H_{0}}\right)^{*}\right|$ is a non-singular strongly cyclic and separating vector for $\pi\left(\mathcal{L}^{\dagger}(\mathcal{D})\right)$ contained in $\sigma_{2}(\mathcal{D})$ and the Gibbs state $\omega_{\varphi}^{\beta}$ is represented as

$$
\omega_{\varphi}^{\beta}(X)=\left(\pi(X) \Omega_{\varphi} \mid \Omega_{\varphi}\right), \quad X \in \mathcal{L}^{\dagger}(\mathcal{D}) .
$$

Furthermore, if $\Omega_{\varphi}^{i t} \mathcal{D} \subset \mathcal{D}$ for all $t \in \mathbb{R}$, then $\sigma_{t}^{\Omega_{\varphi}}:=\Omega_{\varphi}^{i t} X \Omega_{\varphi}^{i t}, X \in \mathcal{L}^{\dagger}(\mathcal{D}), t \in \mathbb{R}$ is a one-parameter group of *-automorphisms of $\mathcal{L}^{\dagger}(\mathcal{D})$, which is induced by the modular automorphism group

$$
\sigma_{t}^{\Omega_{\varphi}}(\pi(X)):=\triangle_{\Omega_{\varphi}}^{i t} \pi(X) \triangle_{\Omega_{\varphi}}^{-i t}, \quad t \in \mathbb{R}
$$


of $\pi\left(\mathcal{L}^{\dagger}(\mathcal{D})\right)$.

Remark If $\bar{T}$ commutes to $e^{-H_{0}}$, that is $e^{-H_{0}} \bar{T} \subset \bar{T} e^{-H_{0}}$, then $\alpha_{t}^{\varphi}(X)=$ $\left|T^{*}\right|^{i t} \sigma_{2 t}^{\varphi}(X)\left|T^{*}\right|^{-i t}$, for all $X \in \mathcal{L}^{\dagger}(\mathcal{D})$ and $t \in \mathbb{R}$. Since $\sigma_{t}^{\varphi}$ is a $*$-automorphism of $\mathcal{L}^{\dagger}(\mathcal{D})$, but $\alpha_{t}^{\varphi}$ is not a $*$-automorphism, these two one-parameter groups $\left\{\sigma_{t}^{\varphi}\right\}$ and $\left\{\alpha_{t}^{\varphi}\right\}$ of automorphisms of $\mathcal{L}^{\dagger}(\mathcal{D})$ have no relation in general.

For the Gibbs state $\omega_{\psi}^{\beta}$ on $\mathcal{L}^{\dagger}(\mathcal{D})$ we similarly have the following

Theorem 5.2.2 Let $\left\{\varphi_{n}\right\}$ be a generalized Riesz system with a constructing pair $\left(\left\{f_{n}\right\}, T\right), n \in \mathbb{N}_{0}$. Suppose that there exists a dense subspace $\mathcal{D}$ in $\mathcal{H}$ satisfying

(i) $e^{-\frac{\beta}{2} H_{0}} \mathcal{H} \subset \mathcal{D} \subset D\left(T^{-1}\right) \cap D\left(\left(T^{-1}\right)^{*}\right)$,

(ii) $\left(T^{-1}\right)^{*}\lceil\mathcal{D} \in \mathcal{L}(\mathcal{D})$,

(iii) $\mathcal{L}^{\dagger}(\mathcal{D})$ is self-adjoint.

Then $\Omega_{\psi}:=\frac{1}{\sqrt{Z_{\psi}}}\left|\left(\left(T^{-1}\right)^{*} e^{-\frac{\beta}{2} H_{0}}\right)^{*}\right|$ is a non-singular strongly cyclic and separating vector for $\pi\left(\mathcal{L}^{\dagger}(\mathcal{D})\right)$ contained in $\sigma_{2}(\mathcal{D})$ and the Gibbs state $\omega_{\psi}^{\beta}$ is represented as

$$
\omega_{\psi}^{\beta}(X)=\left(\pi(X) \Omega_{\psi} \mid \Omega_{\psi}\right), \quad X \in \mathcal{L}^{\dagger}(\mathcal{D})
$$

Furthermore, if $\Omega_{\psi}^{i t} \mathcal{D} \subset \mathcal{D}$ for all $t \in \mathbb{R}$, then

$$
\sigma_{t}^{\Omega_{\psi}}(X):=\Omega_{\psi}^{i t} X \Omega_{\psi}^{-i t}, \quad X \in \mathcal{L}^{\dagger}(\mathcal{D}), t \in \mathbb{R}
$$

is a one-parameter group of *-automorphisms of $\mathcal{L}^{\dagger}(\mathcal{D})$, which is induced by the modular automorphism group

$$
\sigma_{t}^{\Omega_{\psi}}(\pi(X)):=\triangle_{\Omega_{\psi}}^{i t} \pi(X) \triangle_{\Omega_{\psi}}^{-i t}, \quad t \in \mathbb{R}
$$

of $\pi\left(\mathcal{L}^{\dagger}(\mathcal{D})\right)$.

\section{Conclusions}

In this paper we have discussed how to generalize the standard notions of Heisenberg dynamics, Gibbs states, KMS- condition and Tomita-Takesaki theory to the case in which the dynamics is driven by a non self-adjoint Hamiltonian, as it often happens in PT- and in pseudo-hermitian quantum mechanics and we have chosen to consider observables as elements of $\mathcal{L}^{\dagger}(\mathcal{D})$. We have also seen how generalized Riesz systems can be used in this context, and how the results deduced here differ from the standard ones. We have also discussed some preliminary results on entropy and on the TomitaTakesaki theory in our settings.

Of course, many other aspects could be considered in future, from the use of Gibbs states defined by generalized Riesz systems in the analysis of concrete physical systems 
to more mathematical aspects. For instance, since it is often difficult or even impossible to find a common invariant dense domain $\mathcal{D}$ for the observables, one could try to enlarge the setting to some other relevant subset of $\mathcal{L}^{\dagger}(\mathcal{D}, \mathcal{H})$. We plan to work on these and other aspects of our framework soon.

Acknowledgements H. I. is supported by JSPS KAKENHI Grant Number 20K14335. H.I also acknowledges the Daiichi University of Pharmacy. F. B. thanks the University of Tokyo for financial support during a preliminary stage of this research. F. B. also acknowledges the University of Palermo, and the Gruppo Nazionale di Fisica Matematica of Indam. C. T. acknowledges the University of Palermo, and the Gruppo Nazionale di Analisi Matematica la Probabilità e le loro Applicazioni of Indam. Finally, we wish to thank the referee for his/her fruitful comments.

Funding Open access funding provided by Universit'a degli Studi di Palermo within the CRUI-CARE Agreement.

Open Access This article is licensed under a Creative Commons Attribution 4.0 International License, which permits use, sharing, adaptation, distribution and reproduction in any medium or format, as long as you give appropriate credit to the original author(s) and the source, provide a link to the Creative Commons licence, and indicate if changes were made. The images or other third party material in this article are included in the article's Creative Commons licence, unless indicated otherwise in a credit line to the material. If material is not included in the article's Creative Commons licence and your intended use is not permitted by statutory regulation or exceeds the permitted use, you will need to obtain permission directly from the copyright holder. To view a copy of this licence, visit http://creativecommons.org/licenses/by/4.0/.

\section{References}

1. Bender, C.M.: PT Symmetry in Quantum and Classical Physics. World Scientific, Singapore (2019)

2. Mostafazadeh, A.: Pseudo-Hermitian representation of quantum mechanics. Int. J. Geom. Methods Mod. Phys. 7, 1191-1306 (2010)

3. Bender, C., Fring, A., Günther, U., Jones, H.: Special issue on quantum physics with non-Hermitian operators. J. Phys. A 45, 44 (2012)

4. Bagarello, F., Passante, R., Trapani, C.: Non-Hermitian Hamiltonians in Quantum Physics. In: Selected Contributions from the 15th International Conference on Non-Hermitian Hamiltonians in Quantum Physics, Palermo, Italy, 18-23 May 2015. Springer, Berlin (2016)

5. Bagarello, F., Gazeau, J.P., Szafraniec, F.H., Znojil, M. (eds.): Non-selfadjoint Operators in Quantum Physics: Mathematical Aspects. Wiley, Hoboken (2015)

6. Bagarello, F.: Deformed canonical (anti-)commutation relations and non hermitian Hamiltonians. In: Bagarello, F., Gazeau, J.P., Szafraniec, F.H., Znojil, M. (eds.) Non-selfadjoint Operators in Quantum Physics: Mathematical Aspects. Wiley, Hoboken (2015)

7. Bagarello, F., Inoue, A., Trapani, C.: Non-self-adjoint hamiltonians defined by Riesz bases. J. Math. Phys. 55, 033501 (2014)

8. Bagarello, F., Inoue, H., Trapani, C.: Biorthogonal vectors, sesquilinear forms, and some physical operators. J. Math. Phys. 59, 033506 (2018)

9. Bagarello, F., Inoue, H., Trapani, C.: Generalized Riesz systems and quasi bases in Hilbert space Mediterr. J. Math. 17, 41 (2020)

10. Inoue, H., Takakura, M.: Regular biorthogonal pairs and pseudo-bosonic operators. J. Math. Phys. 57, 083503 (2016)

11. Inoue, H., Takakura, M.: Non-self-adjoint hamiltonians defined by generalized Riesz bases. J. Math. Phys. 57, 083505 (2016)

12. Inoue, H.: General theory of regular biorthogonal pairs and its physical operators. J. Math. Phys. 57, 083511 (2016)

13. Inoue, H.: Semi-regular biorthogonal pairs and generalized Riesz bases. J. Math. Phys. 57, 113502 (2016) 
14. Inoue, H.: Ordered structures of constructing operators for generalized Riesz systems. Int. J. Math. Math. Sci. 2018, 3268251 (2018)

15. Bagarello, F., Kuźel, S.: Generalized Riesz systems and orthonormal sequences in Krein spaces. J. Phys. A 53, 8 (2020)

16. Bellomonte, G., Trapani, C.: Riesz-like bases in rigged Hilbert spaces. Z. Anal. Anwend. 35, 243-265 (2016)

17. Kamuda, A., Kuźel, S.: Towards generalized Riesz systems theory. Complex Anal. Oper. Th. 14, 28 (2020)

18. Bagarello, F., Trapani, C., Triolo, S.: Gibbs states defined by biorthogonal sequences. J. Phys. A 49, 405202 (2016)

19. Bebiano, N., da Providencia, J., da Providencia, J.P.: Toward non-Hermitian quantum statistical thermodynamics. J. Math. Phys. 61, 022102 (2020)

20. Antoine, J.-P., Inoue, A., Trapani, C.: Partial *-Algebras and Their Operator Realizations. Kluwer, Dordrecht (2002)

21. Antoine, J.-P., Trapani, C.: Partial Inner Product Spaces. Theory and Applications. Springer, Berlin (2009)

22. Bagarello, F.: Algebras of unbounded operators and physical applications: a survey. Rev. Math. Phys. 19(3), 231-272 (2007)

23. Schmüdgen, K.: Unbounded Operator Algebras and Representation Theory. Birkhäuser-Verlag, Basel (1990)

24. Sakai, S.: Operator Algebras in Dynamical Systems, the Theory of Unbounded Derivations in $C^{*}$ Algebras. Cambrige University Press, Cambrige (1991)

25. Inoue, A.: Tomita-Takesaki Theory in Algebras of Unbounded Operators. Lecture Notes in Mathematics, vol. 1699. Springer, Berlin (1998)

26. Takesaki, M.: Tomita's Theory of Modular Hilbert Algebras and Its Applications. Lecture Notes in Mathematics, vol. 128. Springer, Berlin (1970)

27. Lassner, G., Timmermann, W.: Normal states on algebras of unbounded operators. Rep. Math. Phys. 3, 295-305 (1972)

Publisher's Note Springer Nature remains neutral with regard to jurisdictional claims in published maps and institutional affiliations. 